\title{
A polarimetric investigation of Jupiter: Disk-resolved imaging polarimetry and spectropolarimetry ${ }^{\star}$
}

\author{
W. McLean ${ }^{1,2}$, D. M. Stam ${ }^{3}$, S. Bagnulo ${ }^{1}$, G. Borisov ${ }^{1,4}$, M. Devogèle ${ }^{5,6}$, A. Cellino ${ }^{7}$, J. P. Rivet ${ }^{6}$, P. Bendjoya ${ }^{6}$, \\ D. Vernet ${ }^{8}$, G. Paolini ${ }^{3}$, and D. Pollacco ${ }^{2}$ \\ 1 Armagh Observatory, College Hill, Armagh BT61 9DG, UK \\ e-mail: wmc@arm.ac.uk \\ 2 Department of Physics, University of Warwick, Gibbet Hill Road, Coventry CV4 7AL, UK \\ ${ }^{3}$ Faculty of Aerospace Engineering, Technical University Delft, Kluyverweg 1, 2629 HS Delft, The Netherlands \\ ${ }^{4}$ Institute of Astronomy and NAO, 72 Tsarigradsko Chaussee Blvd., 1784 Sofia, Bulgaria \\ 5 Université de Liège, Space Sciences, Technologies and Astrophysics Research (STAR) Institute, Allée du 6 Août 19c, Sart Tilman, \\ 4000 Liège, Belgium \\ ${ }^{6}$ Université Côte d'Azur, Observatoire de la Côte d'Azur, CNRS, Laboratoire Lagrange, 06304 Nice, France \\ 7 INAF-Osservatorio Astrofisico di Torino, 10025 Pino Torinese, Italy \\ 8 Université Côte d'Azur, Observatoire de la Côte d'Azur, Dept. Galilée, 06304 Nice, France
}

Received 14 July 2016 / Accepted 4 February 2017

\begin{abstract}
Context. Polarimetry is a powerful remote sensing tool to characterise solar system planets and, potentially, to detect and characterise exoplanets. The linear polarisation of a planet as a function of wavelength and phase angle is sensitive to the cloud and haze particle properties in planetary atmospheres, as well as to their altitudes and optical thicknesses.

Aims. We present for the first time polarimetric signals of Jupiter mapped over the entire disk, showing features such as contrasts between the belts and zones, the polar regions, and the Great Red Spot. We investigate the use of these maps for atmospheric characterisation and discuss the potential application of polarimetry to the study of the atmospheres of exoplanets.

Methods. We have obtained polarimetric images of Jupiter, in the $B, V$, and $R$ filters, over a phase angle range of $\alpha=4^{\circ}-10.5^{\circ}$. In addition, we have obtained two spectropolarimetric datasets, over the wavelength range 500-850 nm. An atmospheric model was sought for all of the datasets, which was consistent with the observed behaviour over the wavelength and phase angle range.

Results. The polarimetric maps show clear latitudinal structure, with increasing polarisation towards the polar regions, in all filters. The spectropolarimetric datasets show a decrease in polarisation as a function of wavelength along with changes in the polarisation in methane absorption bands. A model fit was achieved by varying the cloud height and haze optical thickness; this can roughly produce the variation across latitude for the $V$ and $R$ filters, but not for the $B$ filter data. The same model particles are also able to produce a close fit to the spectropolarimetric data. The atmosphere of Jupiter is known to be complex in structure, and data taken at intermediate phase angles (unreachable for Earth-based telescopes) seems essential for a complete characterisation of the atmospheric constituents. Because exoplanets orbit other stars, they are observable at intermediate phase angles and thus promise to be better targets for Earthbased polarimetry.
\end{abstract}

Key words. planets and satellites: atmospheres - radiative transfer - planets and satellites: gaseous planets - polarization

\section{Introduction}

Polarimetry is a proven technique for remote sensing in the solar system for bodies of all sizes. Integrated over the stellar disk, light from most stars can be considered unpolarised (see Kemp et al. 1987), while it can become polarised upon interaction with a planet or another object, such as a moon or asteroid. Polarimetry of solar system bodies can serve both as a method to investigate unknown properties of these bodies and as a benchmark for the study of exoplanets. A famous example of the use of polarimetry in planetary characterisation is the study of the clouds of Venus by Hansen \& Hovenier (1974), who successfully constrained the size and composition of the clouds through

* Based on data obtained with ToPol at the one-metre "Omicron" (West) telescope of the C2PU (Centre Pédagogique Planète et Univers) facility (Calern plateau, Observatoire de la Côte d'Azur, France), and FoReRo2, at the two-metre RCC telescope of the Rozhen National Astronomical Observatory, Bulgaria. fitting theoretical models to ground-based polarimetric observations. Since direct starlight is virtually unpolarised, polarimetry is a promising technique for enhancing the contrast between a star and an exoplanet, hence for direct detection and characterisation of exoplanets through the polarised light they reflect (Wiktorowicz \& Stam 2015).

Jupiter's atmosphere consists of several regions with different cloud composition and altitude, known as belts and zones. The belts are seen as darker bands encircling the planet, whilst the zones are the brighter regions. The zones of Jupiter are thought to have dense ammonia ice clouds at higher altitudes in the atmosphere, whilst the belts of Jupiter have thinner clouds at lower altitudes (Ingersoll et al. 2004). The exact chemical makeup of the particles in the belts and zones is not known, but could be complex compounds of sulphur, phosphorous, and carbon (Ingersoll 1976). Above the ammonia cloud layers, a diffuse layer of haze particles is present. Towards the polar regions, a thick haze covers the planet. The term "haze" is used in this 
study to denote particles with sub-micron radii in optically thin layers, whilst the term "clouds" is used to refer to particles of larger size in optically thicker layers.

There is a considerable amount of observational study of the atmosphere of Jupiter with contributions from both groundbased and Earth-orbiting telescopes taken over several decades. There have been major concentrated bursts of information from the space missions that have encountered the Jovian system, namely Pioneer 10 and 11, the Voyager missions, Galileo, and Cassini. West et al. (1986) review observational results from the Pioneer missions, Voyager, and ground-based studies carried out prior to 1986, as well as theoretical studies carried out up to then. West et al. (2004) give a more updated review of the cloud microphysics of Jupiter, taking recent space missions into account. These authors also present some of the most recent images of Jupiter taken with Cassini, when it flew by for a gravity assist on its way to Saturn in 2000. The images were taken in filters with the strongest methane absorption bands: $619 \mathrm{~nm}, 727 \mathrm{~nm}$, and $750 \mathrm{~nm}$. This was in the second half of the northern hemisphere summer (summer solstice was in May 2000), and thus the second half of the southern hemisphere winter with the images showing an increase in brightness at the south polar hood of Jupiter and in the equatorial band. The south polar hood was bright during that period because of a stratospheric haze layer concentrated at high latitudes at around the 3 mbar pressure level. The north pole also had a bright polar hood, but at the time of the fly-by it was more diffuse and spread over a larger area than the southern polar hood. The equatorial region contains a tropospheric haze layer, at around 200 mbar, which appears to be more dense than in the midlatitude regions. The data from Cassini show that the bright equatorial band is not symmetric around the equator, which might reflect another seasonal effect. The storm region in the southern hemisphere known as the Great Red Spot, with the centre located $22^{\circ}$ south of the equator, is a region of elevated tropospheric haze (West et al. 2004).

Light reflected by Jupiter is usually polarised owing to scattering taking place in the atmosphere. Studying the variation of polarisation across the disk of Jupiter can give constraints on the properties of the different particle types present. The first polarimetric observations of Jupiter were carried out by Lyot (1929); these observations revealed a strong positive value of linear polarisation at the poles of Jupiter of $\approx 5-8 \%$ with the direction of polarisation perpendicular to the limb. Close to the equator, Lyot measured a polarisation of almost zero near opposition and a polarisation of $\approx 0.4 \%$, directed parallel to the equator, for phase angles around $10^{\circ}$. Lyot frequently observed different parts of Jupiter's disk in polarised light between 1922 and 1926, and the polarisation in the polar regions was always higher than that of the centre of the disk. The polarisation in the equatorial region was observed to change direction with distance from the centre of the planetary disk, while the absolute value of polarisation remained small. Other observational studies over the last $50 \mathrm{yr}$ or so have confirmed the measurement by Lyot of the stronger polarisation in the polar regions, reaching around $7-8 \%$ in blue light (Dollfus 1957; Gehrels et al. 1969; Morozhenko \& Yanovitskii 1973; Hall \& Riley 1976; Carlson \& Lutz 1989; Starodubtseva et al. 2002; Shalygina et al. 2008). The most recent study is by Schmid et al. (2011), who carried out imaging polarimetry and spectropolarimetry of Jupiter, showing a relatively high polarisation at the poles, with a maximum of around $11.5 \%$ in the south and $10 \%$ in the north, where spring had just started, for a phase angle near $10.4^{\circ}$.
Earth-based observations of Jupiter are limited to a small phase angle range $\left(0^{\circ} \lesssim \alpha \lesssim 12^{\circ}\right)$, where only low degrees of polarisation are to be expected, because of the near-backward scattering direction (Dlugach \& Mishchenko 2008). The higher polarisation at the limbs is due to a relatively large contribution of highly polarised second order scattered light. In order to gain a wider phase angle coverage for Jupiter and a larger variation in polarisation values, data from space missions are thus required (from Earth, only the inner planets Venus and Mercury can be observed at phase angles ranging from almost zero to almost $180 \mathrm{deg}$ ). The Pioneer 10 and 11 spacecrafts obtained polarisation measurements of Jupiter in the 1970s, using the onboard photopolarimeter carried by each spacecraft. It was found that, for a phase angle of $\approx 90^{\circ}$, the polarisation in the $B$ and $R$ filters reached a level as high as $\approx 50 \%$ at the poles with comparatively lower values $(<10 \%)$ in the equatorial latitudes (Smith \& Tomasko 1984). West et al. (2015) give a more detailed overview of space-based polarimetric studies of Jupiter.

Models of the polarisation of the light reflected by Jupiter were developed by Morozhenko \& Yanovitskii (1973), Mishchenko (1990), Dlugach \& Mishchenko (2008). These studies all attempted to carry out interpretation of measurements of the linear polarisation of the centre of Jupiter's disk in terms of particle refractive index and size distribution, using both Mie theory for spherical particles and also several types of non-spherical particles in the case of Dlugach \& Mishchenko (2008). Ultimately it was concluded that at least some of the haze and/or cloud particles are likely to be non-spherical, and owing to the sensitivity of polarisation to particle properties, observations at phase angles greater than those attainable from Earth were required to provide a quantitative fit. In spite of Pioneer and Galileo observations (the latter covering a much wider phase angle range, but with a more limited spectral coverage), a reliable particle size distribution for Jupiter has not yet been derived. The main scatterers present in the Jovian atmosphere are thought to be ammonia ice crystals, possibly coated with organic haze particles condensing from the stratosphere; these cannot be described by spheres or ellipsoids, especially for the purpose of interpreting polarisation data.

This study presents both disk-resolved imaging polarimetry and spectropolarimetry of Jupiter at seven different epochs. The imaging polarimetry datasets were obtained between February and December 2015 using the Torino Polarimeter (ToPol), at the one-metre "Omicron" (West) telescope of the C2PU (Centre Pédagogique Planète et Univers) facility (Calern plateau, Observatoire de la Côte d'Azur, France), in three broadband filters, $B, V$, and $R$. From these, polarimetric maps were constructed with a spatial resolution on the planet, with an equatorial diameter at the 1 bar pressure level of about $140000 \mathrm{~km}$, of approximately $774000 \mathrm{~km}^{2}$ (about $880 \mathrm{~km} \times 880 \mathrm{~km}$ ) per pixel at the centre of the disk. The spectropolarimetric datasets were obtained in December 2014, and November 2015, using the 2Channel-Focal-Reducer instrument (FoReRo2), equipped with polarimetric optics, at the two-metre telescope of the Bulgarian National Astronomical Observatory, Rozhen, Bulgaria.

This paper is structured as follows: Sect. 2 gives an overview of the terminology used in describing the observed and modelled planetary radiation. Section 3 gives details of the observations. Section 4 explains the data reduction techniques, and then the results of the observations are presented in Sect. 5. Section 6 describes the radiative transfer algorithm and the atmospheric models used to interpret the observations, then in Sect. 7 the model fits to the data are presented, along with some sample results showing what could be observed from Jupiter-like exoplanets. 
Finally, Sect. 8 summarises and concludes the findings of this study.

\section{Description of planetary radiation}

Integrated across the planetary disk, sunlight (starlight) reflected by an orbiting planet (exoplanet) can be fully described by the Stokes parameters, which are expressed in the form of a fourcomponent column vector,

$\boldsymbol{F}(\lambda, \alpha)=\left(\begin{array}{l}F(\lambda, \alpha) \\ Q(\lambda, \alpha) \\ U(\lambda, \alpha) \\ V(\lambda, \alpha)\end{array}\right)$,

with $\alpha$ the planetary phase angle, that is, the angle subtended by the Sun and the observer, as seen from the centre of the planet. The single scattering angle, $\Theta$, is related to the phase angle by $\alpha=180^{\circ}-\Theta$. The parameter $F$ represents the total flux reflected by the planet, $Q$ and $U$ represent the linearly polarised flux, and $V$ represents the circularly polarised flux. The Stokes parameters, $Q$ and $U$, are defined with respect to the planetary scattering plane, that is the plane containing the Sun, planet, and observer. To transform between reference planes, such as from the optical plane of a polarimeter, the following rotation matrix is used (see Hovenier \& van der Mee 1983):

$\boldsymbol{L}(\beta)=\left(\begin{array}{cccc}1 & 0 & 0 & 0 \\ 0 & \cos 2 \beta & \sin 2 \beta & 0 \\ 0 & -\sin 2 \beta & \cos 2 \beta & 0 \\ 0 & 0 & 0 & 1\end{array}\right)$,

where $\beta$ is the angle between the two reference planes measured in an anti-clockwise direction from the old to the new reference plane when looking towards the observer. Alternatively, instead of using $Q$ and $U$, one can describe the linear polarisation using the total degree of linear polarisation, $P_{\mathrm{L}}$, and the position angle, $\chi$, which can be obtained from $Q$ and $U$. This enables a better graphical representation of direction.

The degree of linear polarisation of reflected starlight is defined as

$P_{\mathrm{L}}=\frac{\sqrt{Q^{2}+U^{2}}}{F}$

and is independent of the choice of reference plane. The angle of linear polarisation with respect to the reference plane, $\chi$, is defined as (Hansen \& Travis 1974)

$\chi=\frac{1}{2} \arctan \left(\frac{U}{Q}\right)$.

Details of how the Stokes parameters were calculated from the telescope observations are given in Sect. 4. The degree of circular polarisation is very small for a planet like Jupiter (Kemp \& Wolstencroft 1971), and it will thus be ignored in this study. This can be carried out without introducing significant errors in the calculated values of $F, Q$, and $U$ (Stam \& Hovenier 2005). The incident sunlight on Jupiter is considered to be unpolarised, since integrated over the solar disk, this is the case to a very small error (Kemp et al. 1987).

For spatially resolved signals, we can use the same formalisms, except that the phase angle $\alpha$ is replaced by the local illumination and viewing angles (i.e. at each given location on the planet) $\theta_{0}, \phi_{0}, \theta$, and $\phi$, respectively, as described in more detail in Sect. 6. For each given location on the planet, the angle between the direction to the light source and the observer is still given by $\alpha$.

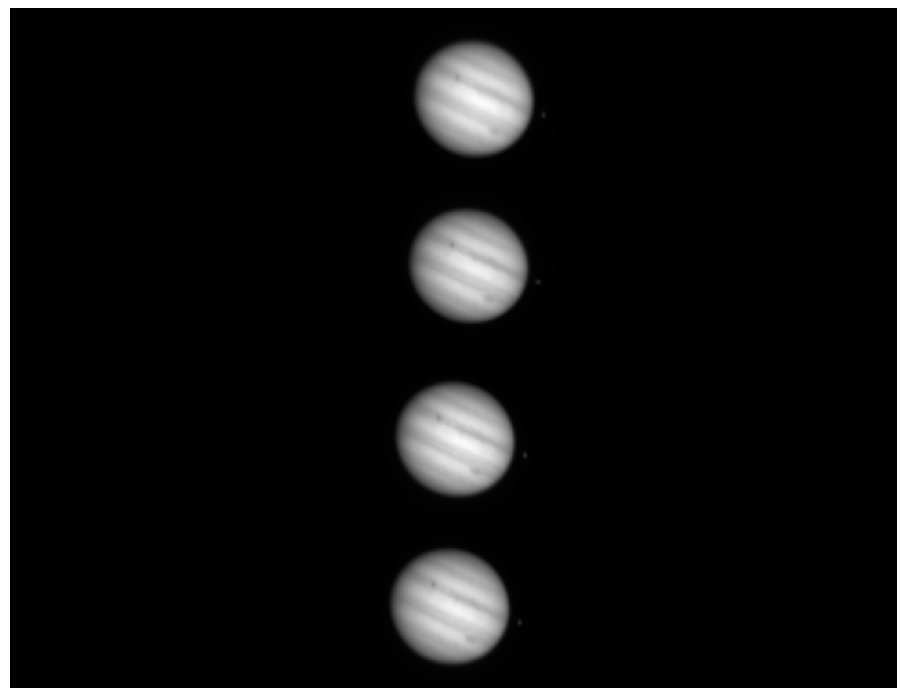

Fig. 1. ToPol CCD image of Jupiter in the $V$ filter. From the top down, the four beams are proportional to $F+Q, F-Q, F+U$, and $F-U$.

\section{Observations}

\subsection{Observing log}

Table 1 shows the log of our observations, which is organised as follows: Col. 1 assigns the name to each dataset, Col. 2 gives the dates of the observations, and Col. 3 the Universal Time (UT) in the middle of each observing block. Column 4 gives the exposure time of an individual frame, in seconds. Column 5 lists the filter used (the two spectropolarimetric observations were taken over the wavelength range $500-850 \mathrm{~nm}$ ). Column 6 then gives the phase angle, $\alpha$, at the time of observation. Columns 7 through 9 give other planetary parameters at the time of observation, namely, angular diameter at the equator in arcseconds, planetary north-pole position angle relative to the north celestial meridian in degrees, and the distance to Jupiter's north pole from the centre of the disk in arcseconds on the sky ${ }^{1}$.

\subsection{Imaging polarimetry with ToPol}

Polarimetric observations of Jupiter were taken in the $B, V$, and $R$ filters at five epochs during 2015. Three epochs have data for all three filters: one epoch for $V$ and $R$ only, and one for just the $V$ filter (see Table 1).

The instrument used for taking these observations is called the Torino Polarimeter (ToPol), and consists of a double wedge Wollaston prism configuration, which is described in detail in Oliva (1997), and the instrument itself is described in Pernechele et al. (2012). Commissioning data are presented in Devogèle et al. (2017). The design of ToPol allows the simultaneous measurement on one CCD read-out as follows: $F+Q$, $F-Q, F+U, F-U$. An example of a raw CCD image of Jupiter is shown in Fig. 1.

In addition to the science frames, bias and dark frames were taken, as well as flatfield images for each filter. Standard stars were also observed to assess the instrumental polarisation and correct for it. The data reduction steps are explained in Sect. 4.1.

\footnotetext{
1 Planetary parameters were calculated using JPL HORIZONS: http://ssd.jpl.nasa.gov/?horizons
} 
Table 1. Observing log.

\begin{tabular}{ccccccccc}
\hline \hline Dataset & Date & UT & Exp. time $(\mathrm{s})$ & Filter or grism & $\alpha\left(^{\circ}\right)$ & Ang. diam. $\left({ }^{\prime \prime}\right)$ & NP ang $\left(^{\circ}\right)$ & NP dist. $\left({ }^{\prime \prime}\right)$ \\
\hline SP1 & $20 / 12 / 2014$ & $03: 30$ & 3.00 & SP & 8.64 & 42.11 & +21.16 & -19.69 \\
\hline IP1 & $25 / 02 / 2015$ & $21: 57$ & 0.05 & $\mathrm{R}$ & 3.94 & 44.74 & +19.23 & -20.92 \\
IP1 & $25 / 02 / 2015$ & $22: 03$ & 0.05 & $\mathrm{~V}$ & 3.94 & 44.74 & +19.23 & -20.92 \\
IP1 & $25 / 02 / 2015$ & $22: 14$ & 0.40 & $\mathrm{~B}$ & 3.94 & 44.74 & +19.23 & -20.92 \\
\hline IP2 & $26 / 02 / 2015$ & $00: 03$ & 0.05 & $\mathrm{R}$ & 3.96 & 44.74 & +19.23 & -20.92 \\
IP2 & $26 / 02 / 2015$ & $00: 13$ & 0.05 & $\mathrm{~V}$ & 3.96 & 44.74 & +19.23 & -20.92 \\
\hline IP3 & $26 / 02 / 2015$ & $21: 13$ & 0.05 & $\mathrm{~V}$ & 4.13 & 44.68 & +19.19 & -20.89 \\
IP3 & $26 / 02 / 2015$ & $21: 24$ & 0.05 & $\mathrm{R}$ & 4.13 & 44.68 & +19.19 & -20.89 \\
IP3 & $26 / 02 / 2015$ & $21: 35$ & 0.20 & $\mathrm{~B}$ & 4.13 & 44.68 & +19.19 & -20.89 \\
\hline IP4 & $17 / 10 / 2015$ & $04: 40$ & 0.05 & $\mathrm{~V}$ & 6.73 & 32.15 & +24.87 & -15.03 \\
\hline SP2 & $06 / 11 / 2015$ & $03: 29$ & 3.00 & $\mathrm{SP}$ & 8.73 & 33.42 & +25.17 & -15.62 \\
\hline IP5 & $10 / 12 / 2015$ & $04: 56$ & 0.08 & $\mathrm{~V}$ & 10.46 & 36.56 & +25.41 & -17.08 \\
IP5 & $10 / 12 / 2015$ & $05: 06$ & 0.50 & $\mathrm{~B}$ & 10.46 & 36.56 & +25.41 & -17.08 \\
IP5 & $10 / 12 / 2015$ & $05: 15$ & 0.07 & $\mathrm{R}$ & 10.46 & 36.56 & +25.41 & -17.08 \\
\hline
\end{tabular}
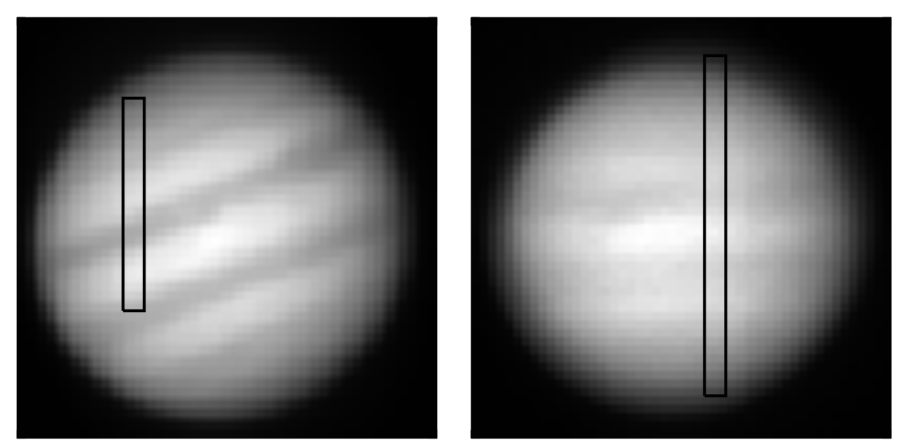

Fig. 2. Left: slit position on Jupiter in December 2014. Right: slit position in November 2015. The reason for the difference in slit height between the two epochs is due to the smaller angular size of Jupiter in dataset SP2, and the slit width was chosen to be narrower in SP2 so that the slit only contained the planet and not the background.

\subsection{Spectropolarimetric observations with FoReRo2}

The spectropolarimetric observations were taken in the wavelength range $500-850 \mathrm{~nm}$ (with a spectral resolution of $2 \mathrm{~nm}$ ) with FoReRo2 at the two-metre telescope of the Bulgarian National Astronomical Observatory, Rozhen, Bulgaria. The instrument is discussed in detail by Jockers et al. (2000) and utilises a retarder Super-Achromatic (in the range 380-790 nm) True Zero-Order Waveplate 5 (APSAW-5) ${ }^{2}$ and a Wollaston prism to measure either $F+Q$ and $F-Q$ or $F+U$ and $F-U$ on each $\mathrm{CCD}$. The retarder waveplate is a recent addition to the instrument and is not described in the original paper by Jockers et al. (2000). The advantage of using the retarder waveplate is that it minimises errors introduced through movement of the instrument and also enables the use of a method known as the beamswapping technique, which is explained in Sect. 4.2. Bias frames were also taken, but no flatfielding was performed. The slit positions of the respective observations are shown in Fig. 2.

\section{Data reduction}

Since there are two distinct types of datasets, this section is subdivided into two: Sect. 4.1 explains the techniques used in the

\footnotetext{
2 http://astropribor.com/content/view/25/33/
}

imaging polarimetry data reduction, and Sect. 4.2 explains those used in the spectropolarimetry data reduction.

\subsection{ToPol data reduction}

The ToPol data reduction required special attention because the individual Stokes parameters can only be derived from combining the images from the four beams (see Fig. 1). As mentioned in Sect. 3.2, the incoming light to the telescope is split into four beams by the double Wollaston prism in the optical setup of the instrument. The CCD is divided into four horizontal strips with each beam projecting an image in one of the strips. The labels $S_{1}$, $S_{2}, S_{3}$, and $S_{4}$, represent the signals (on pixel level) in the individual strips from top to bottom respectively. The reduced Stokes parameters are used in this study, that is $Q$ and $U$ normalised to the total flux of light. For ToPol, the following equations give the reduced Stokes parameters:

$P_{Q}=\frac{Q}{F}=\frac{S_{1}-S_{2}}{S_{1}+S_{2}}$

$P_{U}=\frac{U}{F}=\frac{S_{3}-S_{4}}{S_{3}+S_{4}}$

where $S_{1}=F+Q, S_{2}=F-Q, S_{3}=F+U$ and $S_{4}=F-U$. The errors in these values vary with the $\mathrm{S} / \mathrm{N}$ of the data, and are given by

$$
\begin{gathered}
\sigma_{Q}=\frac{1}{\sqrt{S_{1}^{2}+S_{2}^{2}}} \\
\sigma_{U}=\frac{1}{\sqrt{S_{3}^{2}+S_{4}^{2}}}
\end{gathered}
$$

and

$\sigma_{P}=\sigma_{Q}=\sigma_{\chi}$

since $\sigma_{Q} \approx \sigma_{U}$ (Bagnulo et al. 2009). These errors only hold if there are no alignment and/or distortion errors, and this is discussed in Sects. 4.1.2 and 4.1.3. 
$P_{Q}$

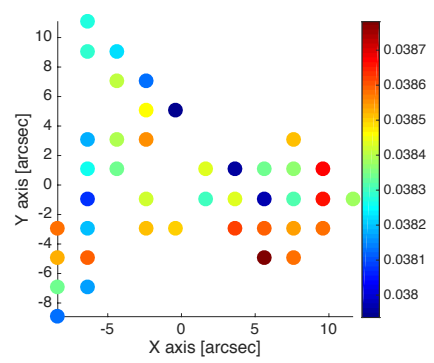

$P_{U}$

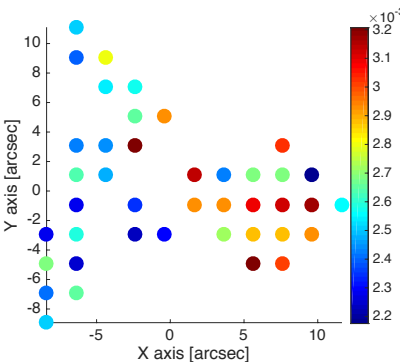

Fig. 3. Left: map of the variation on the CCD of $P_{Q}$. Right: corresponding variation of $P_{U}$.

The different optical paths of the beams lead to two effects that we have to correct for. Firstly, different images on the CCD have to be aligned to be superimposed. Secondly, different images on the CCD have a different level of sharpness, even when taken at the same time. Section 4.1.1 discusses the problems associated with instrumental response, Sect. 4.1.2 explains how the image alignment was carried out, and Sect. 4.1.3 describes the distortion problems and the seeing limitation in detail.

\subsubsection{Instrumental response}

For extended sources it is important to know the instrumental polarisation and its variation across the field of view of the polarimeter. Jupiter was always observed at the centre of the field of view, and is seen as a circle of approximately 100 pixels radius (depending on the apparent size of the planet).

The variation of the instrumental polarisation across the field of view was characterised by observing unpolarised standard stars at different locations in the field of view. Figure 3 represents a map of the variation of the instrumental polarisation around the location of the centre of Jupiter on the CCD. The observed variation in $P_{Q}$ and $P_{U}$ is of the order $10^{-3}$ at most. This variation is negligible compared to the other sources of error, such as photon noise and the error from image alignment (see Sect. 4.1.2), and hence we ignore these small variations in the instrumental polarisation across the CCD.

\subsubsection{Image alignment}

The four strips in each individual image are misaligned owing to the different optical path of the signal in each strip. Additionally, successive images taken are misaligned and this is due to slightly inaccurate guiding of the telescope. Conventional image alignment algorithms, such as those used by IRAF, are optimised for use with point sources, but Jupiter is of course an extended object and not even a precise circle. Some time was spent devising the best method; each method was tried several times by different people with different software.

The first method of aligning the images was by using the phot task in the IRAF daophot package, but this proved to be unreliable, most likely since Jupiter is an extended source with no obvious centre, and is not perfectly circular, so the software was unable to find the centre to a sub-pixel accuracy. The second method again involved using IRAF, but this time using dataset IP3 (see Table 1), where two of the Galilean moons are visible. This also did not prove successful, since the $\mathrm{S} / \mathrm{N}$ of the moons is very low and their images change shape between exposures because of the low signal. A third method involved taking the best alignment from IRAF and then adjusting by eye, but this

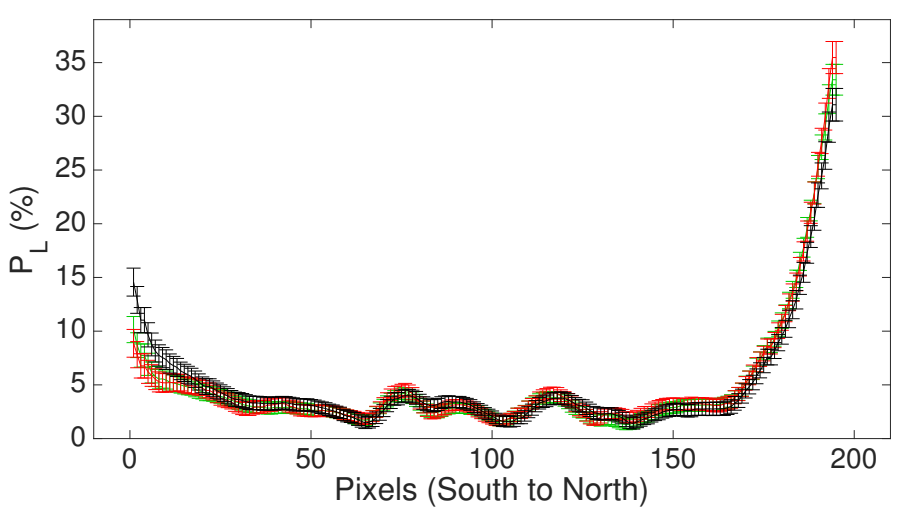

Fig. 4. Plots of $P_{\mathrm{L}}$ vs. distance along Jupiter's central meridian, as derived from three different CCD images read out successively in the $B$ filter, represented by different colours, along with error bars.

obviously is not a reliable method and one cannot get sub-pixel accuracy using this. The results of this method are shown in Fig. 4 for latitudinal cuts along the central meridian of Jupiter, from three successive CCD images in the $B$ filter in dataset IP3, taken seconds apart. The three plots show a slight difference across the centre of the disk and a significant variation at the poles. The differences between the images cannot be accounted for by the error on the flux counts owing to photon noise, and certainly cannot be real since the images were taken a matter of seconds apart. Therefore the difference in $P_{\mathrm{L}}$ at the poles must be due to errors in the alignment of the images on each CCD image. Various MATLAB and IDL routines were trialled for aligning the images, but no success was found with any known image aligning or fitting techniques.

We therefore devised a new aligning technique using $I D L$. This worked by defining an annulus to the outer region of Jupiter (where the signal is lower) and fitting a circle with the centre and radius returned by the algorithm. The next step was to shift each image to a common centre and then cut out and combine to obtain the intensity and polarimetric maps. For each observation, the images were normalised to their maximum value ${ }^{3}$ before they were inputted into the centring algorithm to ensure the same intensity range was used for each strip. Figure 5 shows three successive latitudinal profiles in the $B$ filter in IP3, produced from the same images used to create the plots in Fig. 4. Unlike in Fig. 4, the polarisation at Jupiter's poles is much more consistent between successive images and the features across the centre of the disk are aligned much better. Interestingly, each method gives consistent results for the north pole, but with the new method the polarisation is significantly lower. Given that the differences are all within the error bars for the IDL alignment method, this technique was deemed to be the most reliable and we have thus used this new alignment method for our data analysis.

In order to quantify the remaining sub-pixel errors due to the alignment method, we constructed maps of $P_{Q}, P_{U}, P_{\mathrm{L}}$, and $\chi$ for each Jupiter image, and then the difference between successive images was calculated (a "null" map). The purpose of this exercise was to gain an understanding of the variation of the subpixel alignment error in the six images that were to be combined to form the final image. The final error used is the combined error from both the photon noise and the image alignment.

\footnotetext{
3 These modified images were temporary and created purely for the purpose of aligning the original images to a common centre; the original images were used for calculating the Stokes parameters.
} 


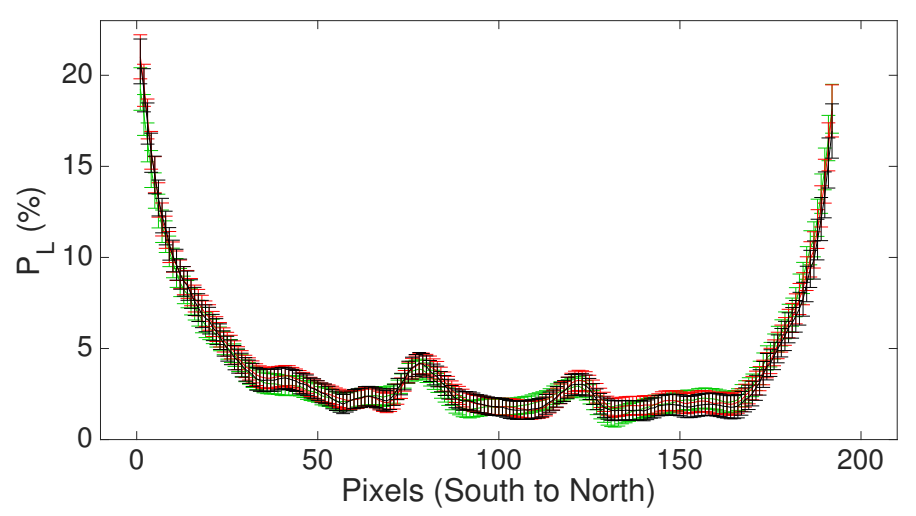

Fig. 5. Same as for Fig. 4, but using the new alignment method.

\subsubsection{Distortion and seeing limitation}

The minimum resolvable detail on the disk of Jupiter is determined by the seeing at the time (calculated by measuring the width of the PSF of a standard star). The plate scale of the images was 0.2379 arcsec/pixel, so to account for the seeing limitation, a $5 \times 5$ box-car smoothing (floating average) was applied to each of the flux images before they were combined to create the Stokes parameters.

The analyser used in ToPol consists of two Wollaston prisms that are sealed together and split the incoming light from the telescope into four beams. The four beams all travel along a slightly different optical path towards the CCD and are focussed at different distances from the CCD. A technique aiming to correct for the distortion was devised, which involved taking an image of a globular cluster and measuring the distances between individual stars in each strip. One strip was used as a reference with the correction applied to the other three. The high number of distances extracted from the cluster image was used to create a reliable map of the distortion across the CCD. An assumption here is that the aberrations are not time dependent. After computing the relative distance of the stars between each other, the residuals of the data were used to create a fit over the entire CCD to obtain error values for every pixel in the two coordinates of the CCD. The residuals were created by comparing distances in three frames with respect to one arbitrarily chosen reference frame. The fitting was performed using a 5th order two-dimensional polynomial and a weight matrix was used in order to give more reliability to data close to the frame centre to produce more accurate results in that region. The order of the polynomial proved to maintain the dispersion of the correction at the 0.1 pixel level in both $x$ and $y$ coordinates, thus using a higher order was deemed to be unnecessary. The corrections obtained from this method were then applied to the Jupiter data on the three strips selected.

However, it was found that this method tended to introduce more errors than it corrected for, as illustrated by Fig. 6. This figure shows a map produced using the distortion correction method compared with a map produced with our own shifting method. These maps are differences between polarimetric maps produced with successive images, taken a matter of seconds apart, so any differences in the polarisation would be due to the alignment method. The residuals are clearly higher for the distortion correction method, thus it was decided not to use this and to produce just polarimetric maps with our shifting method.

\subsection{FoReRo2 spectropolarimetry}

The FoReRo2 instrument has a polarimetric module that allows the use of the beam swapping technique, which is described in Bagnulo et al. (2009), with the key concepts and equations given here. The data reduction for the FoReRo 2 spectropolarimetry was carried out with IRAF with the flux combinations to calculate the Stokes parameters carried out in MATLAB. The science frames were bias subtracted and then several frames were combined for each retarder angle in order to increase $\mathrm{S} / \mathrm{N}$. The combined frames were then each collapsed into one-dimensional spectra and a wavelength calibration was performed using arclamp spectra taken just after the observations. The fluxes of the ordinary and extraordinary beams were then extracted and the beam-swapping technique was applied to calculate the Stokes parameters.

Observations of Jupiter were taken at four retarder waveplate angles: $0^{\circ}, 22.5^{\circ}, 45^{\circ}$, and $67.5^{\circ}$. The beam-swapping technique minimises the errors that can be introduced by instrumental polarisation. The reduced Stokes parameters are calculated as follows (Bagnulo et al. 2009):

$P_{X}=\frac{1}{2 N} \sum_{j=1}^{N}\left[\left(\frac{f^{\|}-f^{\perp}}{f^{\|}+f^{\perp}}\right)_{\alpha_{j}}-\left(\frac{f^{\|}-f^{\perp}}{f^{\|}+f^{\perp}}\right)_{\alpha_{j}+\Delta \alpha}\right]$

where $X$ stands for either $Q$ or $U, N$ represents the number of pairs of retarder angles, $f^{\perp}$ is the flux in the perpendicular beam, $f^{\|}$is the flux in the parallel beam, $\alpha$ is the retarder angle with respect to its zero point in degrees, and $\Delta \alpha$ is $45^{\circ}$. The sum is over all pairs of retarder angles over which the observations have been obtained; in our case, $N=1$.

\section{Observational results}

\subsection{Spectropolarimetry}

Figure 7 shows the degree of linear polarisation, $P_{\mathrm{L}}=\left(P_{Q}^{2}+\right.$ $\left.P_{U}^{2}\right)^{1 / 2}$, versus the wavelength for the two epochs at which Jupiter was observed in the spectropolarimetric mode. Because the frames for each retarder angle were collapsed in IRAF before we combined them to compute the Stokes parameters, these spectra represent sort of averages across the slit (see Fig. 2, for the slit positions on the planet during the two measurements): not only an average across the vertical slit direction, but also across the horizontal direction (even though it is smaller than the vertical direction). The decrease in continuum polarisation in both sets of spectropolarimetric data with increasing wavelength is mostly due to the decrease of Rayleigh scattering and, hence, the increase in contribution by scattering from cloud and haze particles. The difference between the two spectropolarimetry datasets is mostly due to the different atmospheric parameters across the regions sampled in each dataset and the difference in the area covered by the slit. The gaseous absorption bands of methane are mostly absent in the polarisation spectra, except for a band around $620 \mathrm{~nm}$ in the November 2015 data and a band around $720 \mathrm{~nm}$ in both datasets. Both spectropolarimetry datasets appear to be affected by fringing at wavelengths longer than $750 \mathrm{~nm}$.

In the methane bands, the degree of polarisation appears to be lower than in the surrounding continuum. Usually, one expects the degree of polarisation in an absorption band to be higher than that in the continuum because with increasing absorption, the amount of multiple scattered light in the reflected light signal decreases. Since multiple scattering tends to decrease polarisation, absorption bands, such as those of methane, 

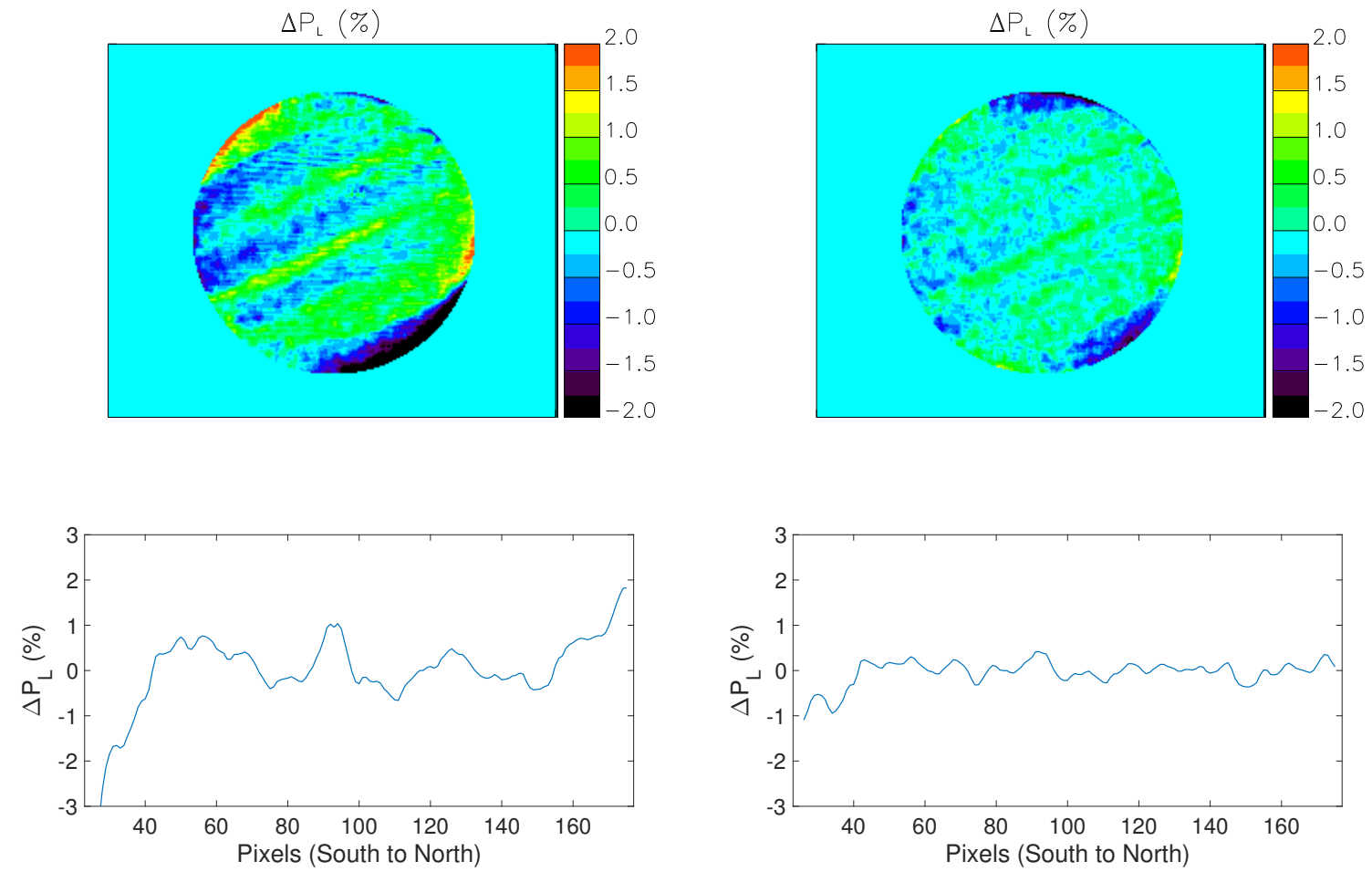

Fig. 6. Top left: $\Delta P_{\mathrm{L}}$ between polarimetric maps made from images taken several seconds apart, using the distortion correction method. Top right: $\Delta P_{\mathrm{L}}$ of maps produced from the same images, but with our shifting method and no distortion correction. Bottom left: $\Delta P_{\mathrm{L}}$ across Jupiter's central meridian of the maps produced with the distortion correction. Bottom right: $\Delta P_{\mathrm{L}}$ across Jupiter's central meridian of the maps made without the distortion correction.

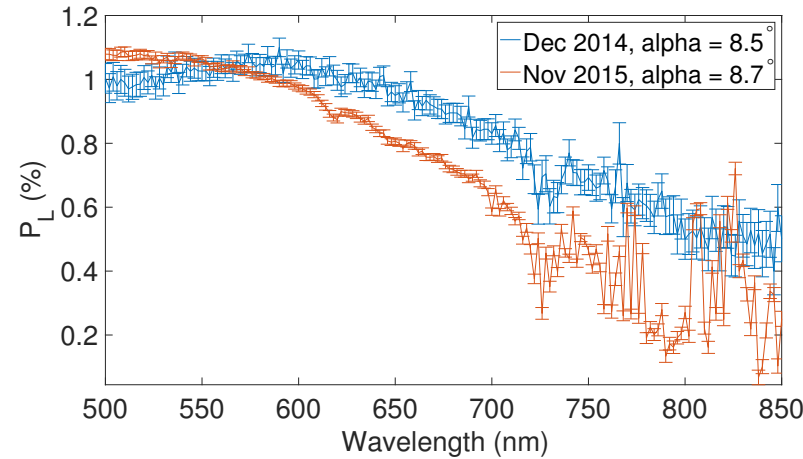

Fig. 7. Spectropolarimetry of Jupiter for the two epochs averaged across both the vertical slit direction and across the horizontal direction.

would thus show up as peaks superimposed on the continuum (Stam et al. 1999, 2004). This effect can indeed be seen in Fig. 4 of Schmid et al. (2011).

However, if the planetary atmosphere is vertically inhomogenous, it is possible for the degree of polarisation in absorption bands to be similar to or lower than that in the continuum (Stam et al. 1999, 2004). This effect arises because with increasing absorption, the observed reflected sunlight has been scattered in increasingly higher atmospheric layers. If the degree of polarisation from scattering by gases and particles in high atmospheric layers is lower than that from (multiple) scattering by gases and particles in the lower layers at the given phase angle, the polarisation can indeed disappear or decrease across (parts of) an absorption band. Figure 3 of Schmid et al. (2011) actually shows this variation of band strength in polarisation in observations of the polarisation ${ }^{4}$ in the continuum (at $600 \mathrm{~nm}$ ) and in

\footnotetext{
4 Schmid et al. (2011) show $Q / I$, with $I$ the total flux.
}

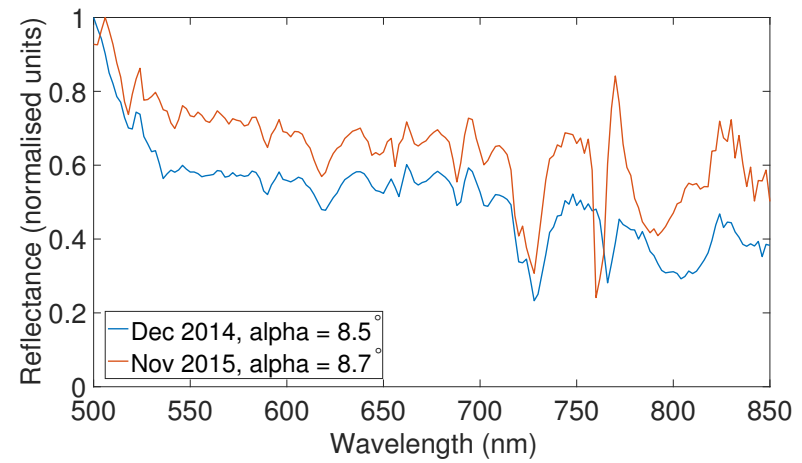

Fig. 8. Normalised reflectance spectra for the two datasets.

a deep methane absorption band (at $887 \mathrm{~nm}$ ) along the central meridian of Jupiter. At latitudes near the south pole and at midlatitudes (the regions indicated by $\mathrm{S}$ - and $\mathrm{N}-$ ), the band polarisation is higher than the continuum polarisation in an absolute sense; the sign of the polarisation only indicates the direction of polarisation. At northern latitudes (region $\mathrm{N}+$ ) and some southern latitudes, however, the band polarisation is below that of the continuum (up to $3 \%$ ) or equal to that of the continuum, and the absorption band shows up as a dip in the continuum polarisation or not at all.

The corresponding reflectance spectra for both of our spectropolarimetry datasets are shown in Fig. 8. These were obtained by dividing the flux of Jupiter by that of a solar analogue, taken with the same instrumental setup. Correspondences between the slopes in both the flux and polarisation can be seen along with the differences in the shapes and depths of the absorption bands. The polarisation spectra appear to be more sensitive to the differences in the depths of the methane absorption bands between 

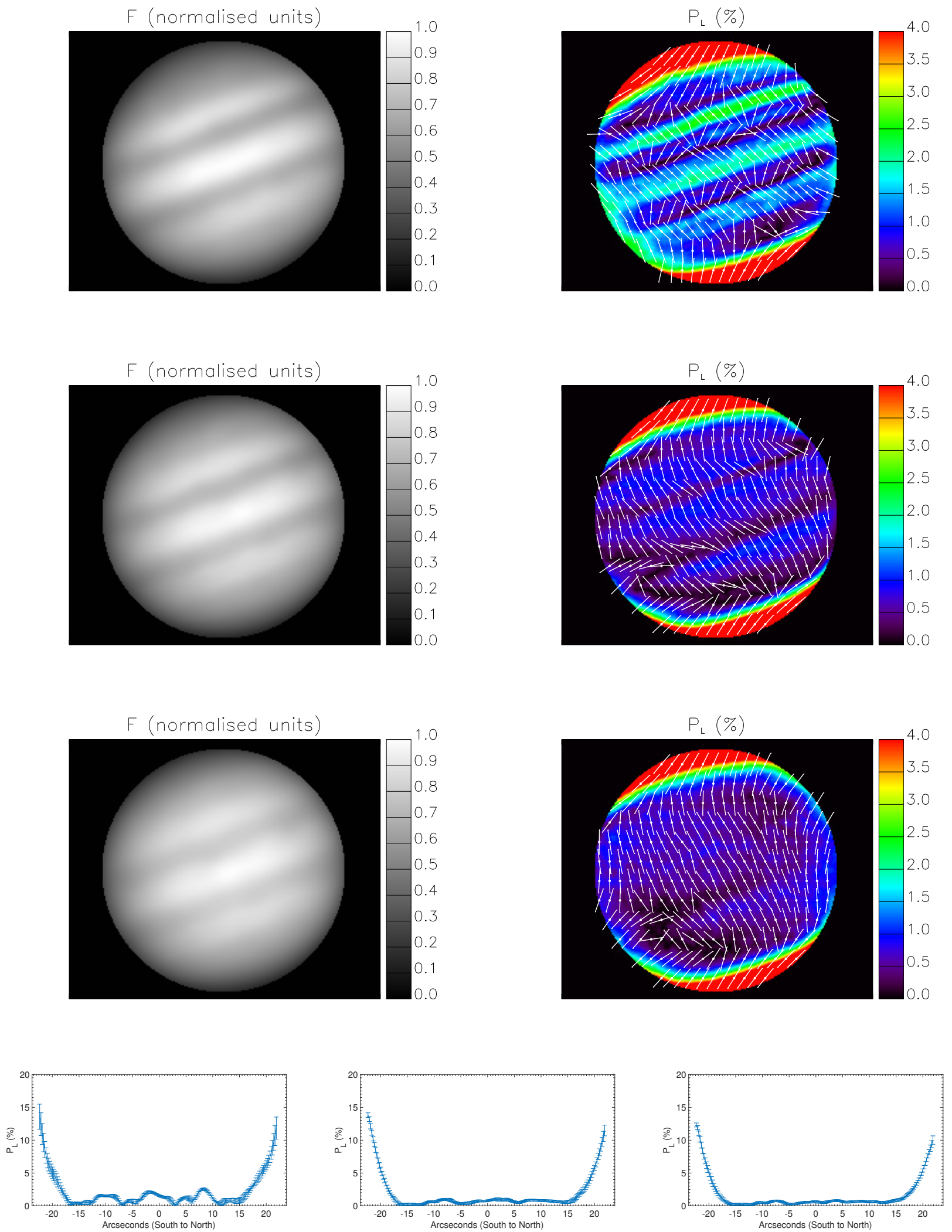

Fig. 9. Dataset IP1. From top to bottom: maps in $B, V$, and $R$ with the bottom plots showing from left to right the polarisation across Jupiter's central meridian in $B, V$, and $R$.

the two epochs. The two datasets were taken almost a year apart and sampled two different regions, so the difference in the absorption feature strength could be due to differences in local time, seasons, and the atmospheric composition in the sampled areas.

\subsection{Imaging polarimetry}

The imaging polarimetry data were taken at five separate observing epochs and phase angles with one dataset near the maximum phase angle observable from Earth, at $10.46^{\circ}$ (the maximum is 
W. McLean et al.: A polarimetric investigation of Jupiter: Disk-resolved imaging polarimetry and spectropolarimetry
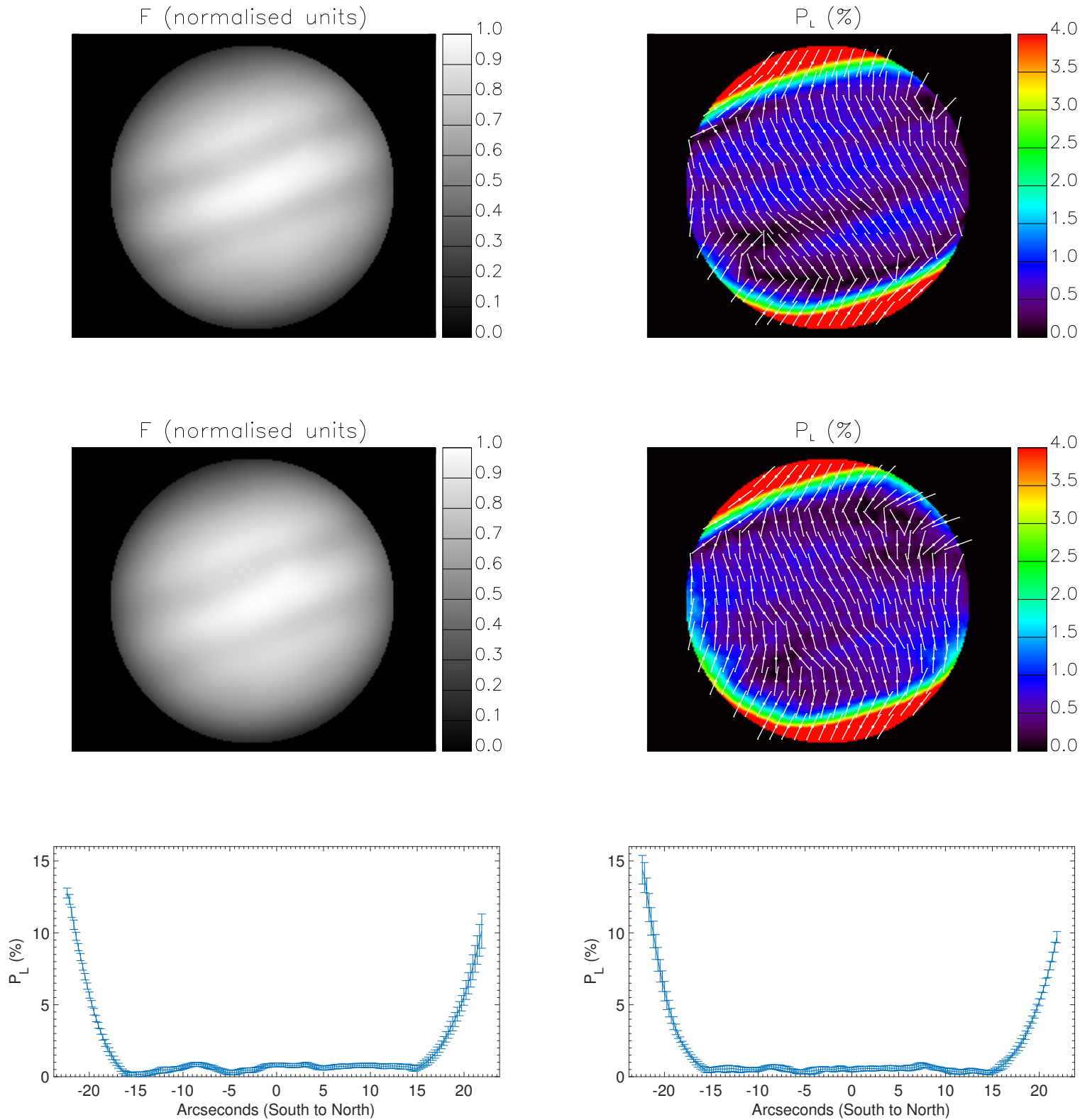

Fig. 10. Dataset IP2. From top to bottom: maps in $V$ and $R$ with the bottom plots showing from left to right the polarisation across Jupiter's central meridian in $V$ and $R$.

about $12 \mathrm{deg}$ ). There are several differences in the planetary parameters of Jupiter at the five epochs, such as the angular diameter of the disk on the sky and, subsequently, the distance to the north pole of the planet from the centre of the disk. The north pole angle of Jupiter also varies slightly relative to that of the north celestial meridian. These parameters are all listed in Table 1.

The Jupiter maps are shown in Figs. 9-13 and are organised as follows: row one shows the $B$ filter, row two $V$, and row three $R$ (except for datasets IP2 and IP4, where data were only taken in the $V$ and $R$, and the $V$ filter, respectively); the left column shows the total flux map and the right column shows the map of $P_{\mathrm{L}}$, with direction arrows indicating the polarisation position angle. The arrows have no arrowhead because the direction of polarisation only varies from $0^{\circ}$ to $180^{\circ}$ and angle $0(180)^{\circ}$ is taken to be the vertical direction. A position angle for each pixel is not shown, as this would clutter the image, so a "rebinned" position angle is shown for sets of $10 \times 10$ pixels on the image; this corresponds to an area of $5.66 \mathrm{sq}$. arcsec, equalling an average area of approximately $7.74 \times 10^{6} \mathrm{~km}^{2}$ on Jupiter. The $P_{\mathrm{L}}$ maps have the scale chosen such that it saturates at $4 \%$ to show the variation in polarisation at lower latitudes in more detail, since for each dataset the polarisation drastically increases towards the polar regions. The bottom plots for each figure show the polarisation across the central meridian of Jupiter, as taken from the polarisation images in the different filters. This value was calculated by taking the values of $P_{\mathrm{L}}$ for the central six longitudes and averaging these values.

\subsubsection{Intensity maps}

From polarimetric observations, usually one also obtains the flux of the object. The flux maps are not calibrated, so what we discuss in the following are relative differences between different filters. The total flux images (Figs. 9-13) clearly show the familiar banded structure of Jupiter. In particular, the north and 

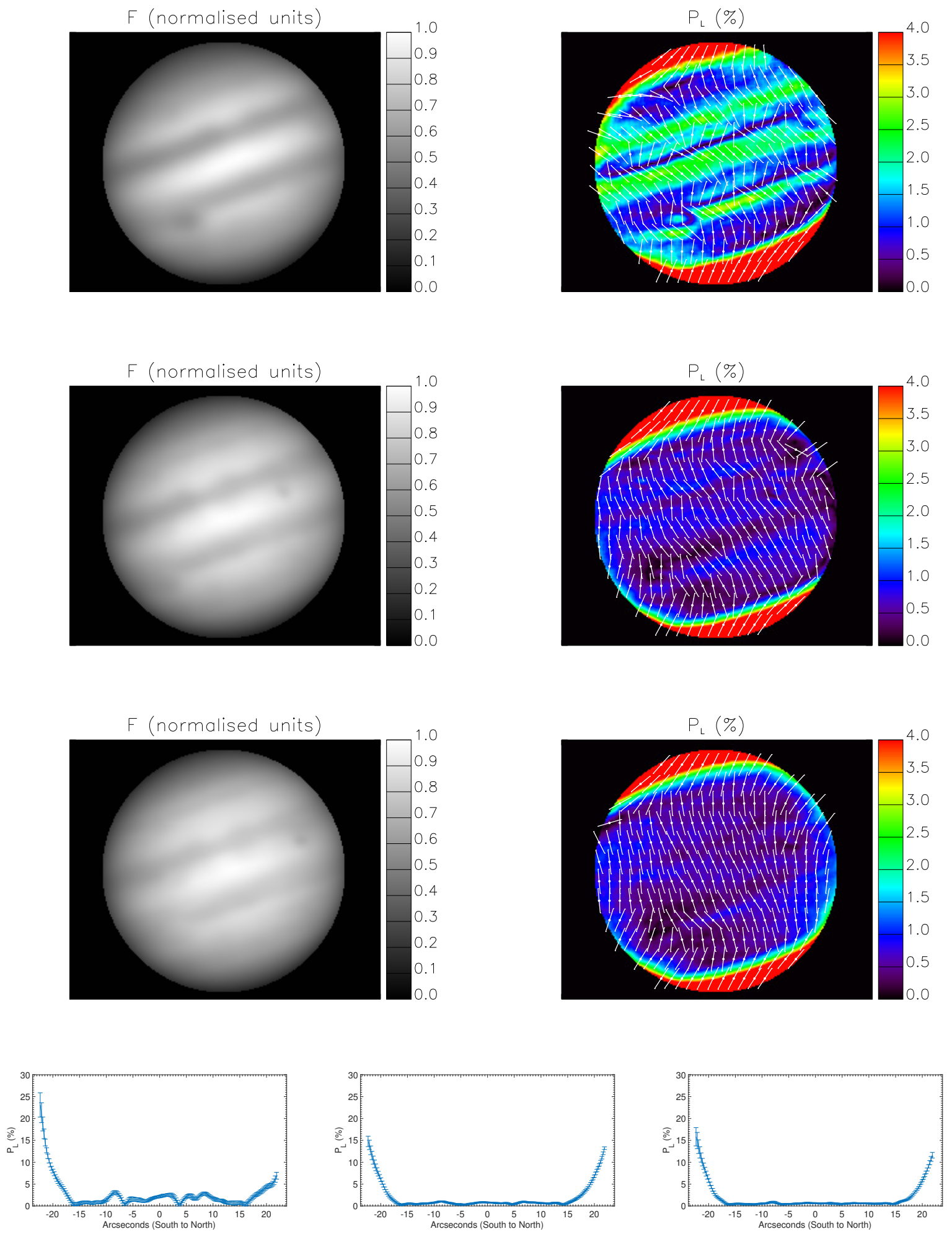

Fig. 11. Dataset IP3. From top to bottom: maps in $B, V$, and $R$ with the bottom plots showing from left to right the polarisation across Jupiter's central meridian in $B, V$, and $R$.

the south equatorial belts stand out as dark stripes between latitudes of about 8 and 20 deg on both sides of the brighter equatorial zone. North and south of the equatorial belts, the planet is brighter across the temperate zones. The narrow, darker belts that are usually observed in these zones are not obvious; separate belts can only be distinguished in dataset IP5 (Fig. 13; see Table 1). These are also visible in dataset IP3, but are not as obvious. Although belts on Jupiter are known to temporarily disappear from view because of overlying hazes, their visibility in our images appears to be related to the viewing conditions. The temperate zones darken slightly towards the poles and transit at roughly $50 \mathrm{deg}$ into the north and south polar regions. A comparison along the planet's limb shows that the total fluxes across the polar regions are lower than can be explained by the large 

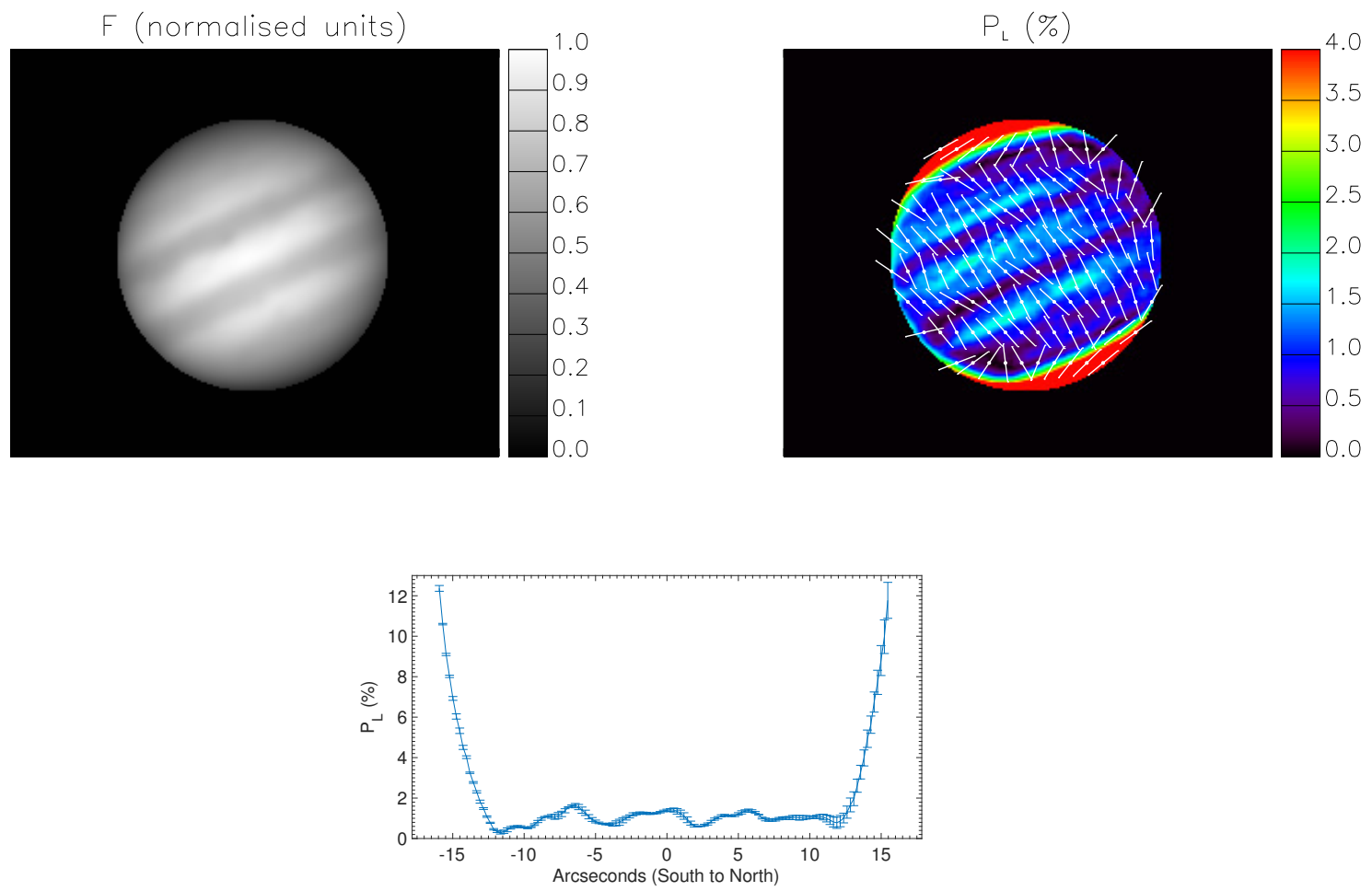

Fig. 12. Dataset IP4. Maps are shown for the $V$ filter with the bottom plot showing the polarisation across Jupiter's central meridian.

local illumination and viewing angles. Indeed, the hazes that are known to cover the polar regions appear to be responsible for the observed low total fluxes.

The contrast between the different belts and zones of Jupiter appears to be greater in the $B$ filter. This is due to greater sensitivity to different cloud altitudes at bluer wavelengths than at redder wavelengths. The clouds in the belts are at lower altitudes and have more gas above them than the clouds in the zones, which reside at higher altitudes. In the blue, the Rayleigh scattering optical thickness is highest, hence the top altitude of the cloud should influence the contrast more. In the red, the gas scattering optical thickness is smallest and hardly influences the light that is reflected by the planet. In the red, the subdued contrast between the belts and the zones should thus mostly be due to differences in cloud composition. In Sect. 6, these influences are investigated in more detail.

The photometric contrast of the belts and zones of Jupiter has been discussed in numerous works (West et al. 1986, 2004) and these works show differences in the clouds and hazes. Aside from in situ measurements taken with the Galileo probe (Sromovsky \& Fry 2002; Young 2003; Vasavada \& Showman 2005), any information on the Jovian atmosphere has come from remote imaging and spectroscopic measurements. The vertical structure of the clouds can be probed by measuring the reflectance in regions of the spectrum where the gas absorption limits the depth that photons can penetrate. In the visible region of the spectrum, methane is the strongest absorber, so methane bands between $600 \mathrm{~nm}$ and $1000 \mathrm{~nm}$ have been used extensively (West et al. 2004).

In datasets IP3 (Fig. 11) and IP5 (Fig. 13), the Great Red Spot (GRS) is in sight. In the GRS, which itself is located on the southern edge of the south equatorial belt, the cloud top altitude is known to be higher than in the surrounding areas. The GRS clearly stands out as a dark region in the $B$-filter images, while it is only slightly brighter than its surroundings in the $R$ filter. The dark dots on Jupiter in IP3 (Fig. 11) and IP5 (Fig. 13) are the shadows of Io and Europa, respectively.

\subsubsection{Polarimetric maps}

In polarisation, our images of Jupiter show differences between the belts and zones; in particular, the polar caps show up clearly despite the small phase angles where the polarisation is usually low. The polarisation of the polar caps appears to be fairly independent of the wavelength, showing relatively high values in the $B, V$, and $R$ bands. In the $B$ band, the polarisation across the belts is higher than that across the zones, while in the $V$ band, the polarisation across the belts is lower than that across the zones. In the $R$ band, much less spatial structure is visible, except for dataset IP5 (Fig. 13), which could be due to the slightly better seeing conditions and, hence, less smearing out of the features.

The polarisation in the GRS stands out in the $B$ band (in datasets IP3 (Fig. 11) and IP5 (Fig. 13)): it is clearly higher than in surrounding regions. In dataset IP5, the direction of polarisation in the GRS differs from that of the surrounding area. Also in dataset IP5, there is a region of relatively high polarisation present just south of the GRS in the $B$ band and the polarisation in the GRS appears to be lower in the $V$ and $R$ bands. It is clear from the polarimetric maps and the latitudinal plots that the polarisation varies considerably between the belts and zones and that this variation changes with filter as well. These variations are likely connected to the particle type and the haze and cloud optical thickness and height; these variations also manifest themselves in the angle of polarisation, which is also observed to change between the different belts and zones and between filters.

The arrows showing the position angle of the polarisation indicate a change across the disk of Jupiter, owing to perhaps different particle types, shapes, and concentrations. Also, the position angle seems to vary with wavelength and time; the latter is possibly indicative of seasonal variations in 

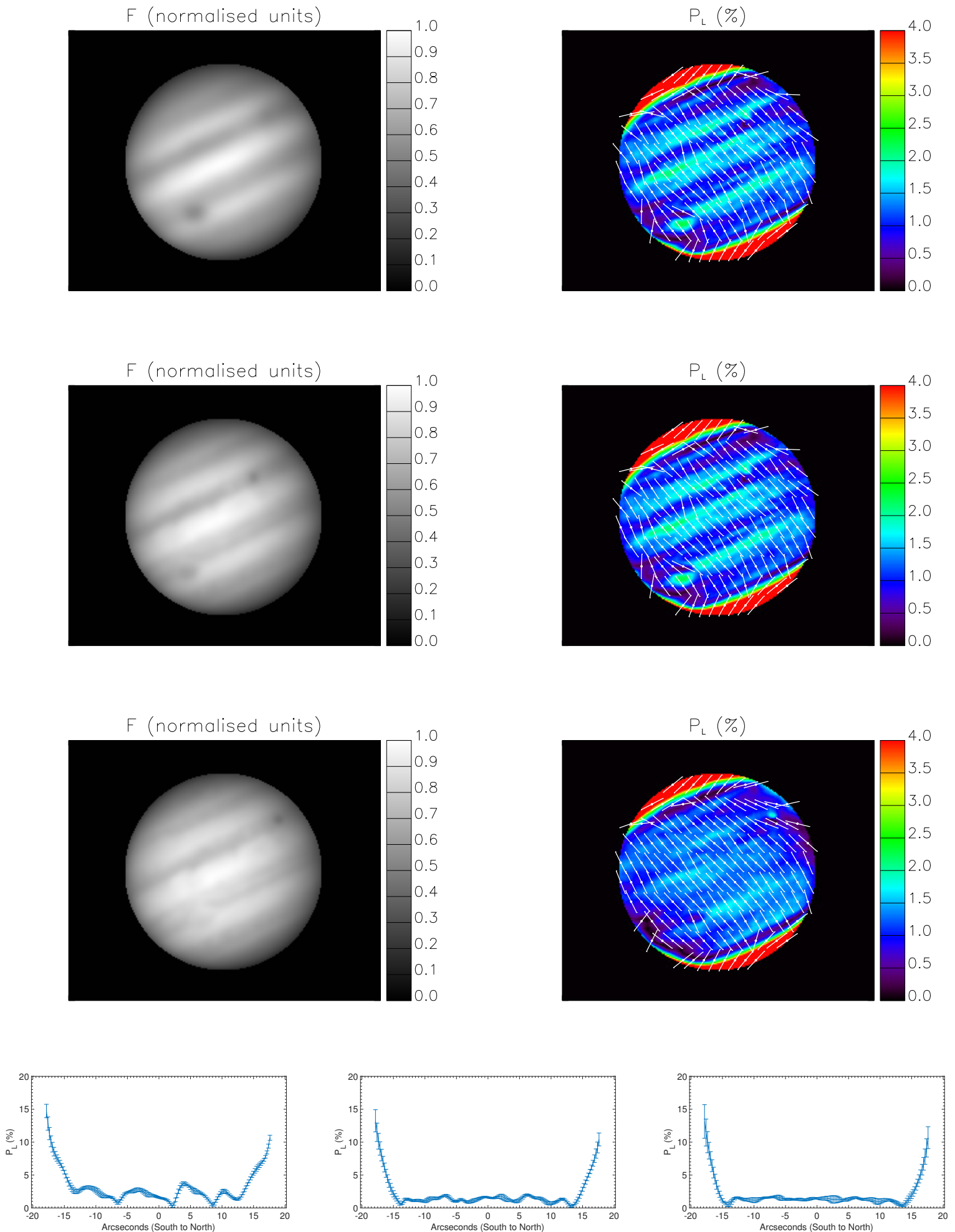

Fig. 13. Dataset IP5. From top to bottom: maps in $B, V$, and $R$ with the bottom plots showing from left to right the polarisation across Jupiter's central meridian in $B, V$, and $R$.

the Jovian atmosphere. Previous studies (Lyot 1929; Dollfus 1957; Gehrels et al. 1969; Morozhenko \& Yanovitskii 1973; Hall \& Riley 1976; Carlson \& Lutz 1989; Starodubtseva et al. 2002; Shalygina et al. 2008; Schmid et al. 2011) have mostly shown a polarisation around the equator that is parallel to the scattering plane, and the polarisation in the polar regions usually perpendicular to the scattering plane. Some of our data is consistent with this, and mostly shows a polarisation that is perpendicular to the scattering plane in the polar regions. However, this is the first study that has presented a detailed evaluation of the position angle across the entire disk for five epochs within a relatively short time period (ten months), so it is difficult to draw conclusions from a comparison with earlier, much less spatially resolved data. The direction of polarisation changes with wavelength and also appears to differ between the belts and zones, in particular, in the $B$ band. The direction of polarisation 
W. McLean et al:: A polarimetric investigation of Jupiter: Disk-resolved imaging polarimetry and spectropolarimetry
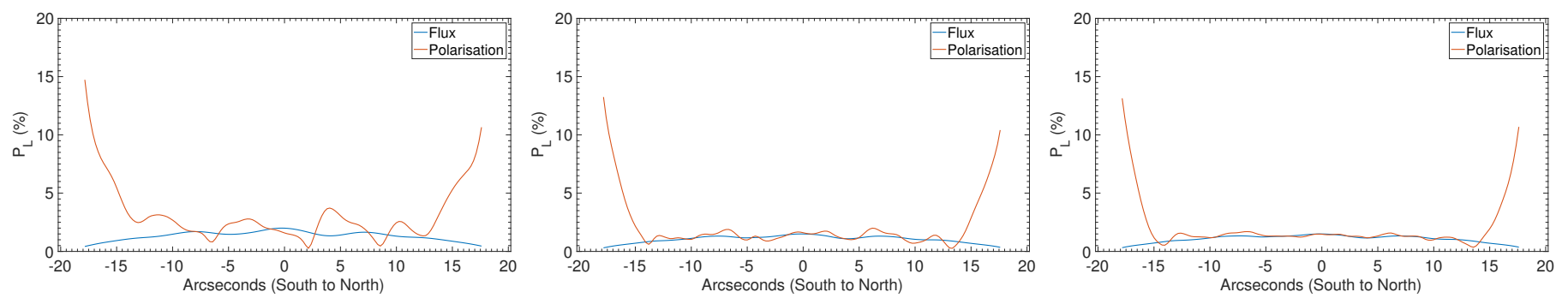

Fig. 14. From left to right: latitudinal plots of the linear polarisation from the central meridian of IP5 in $B, V$, and $R$. The flux values are overplotted in arbitrary units.

at the poles also has a distinct direction compared to the direction on other locations of the disk. Future work will analyse the variation of position angle in the different regions of Jupiter, as a function of both time and wavelength, in more detail.

The bottom plots of Figs. 9-13 show the polarisation across the central meridian of Jupiter, as taken from the polarisation images. These plots all show the variation in the polarisation associated with the belts and zones: the decrease and inversion of the variation up to the midlatitudes with increasing wavelength, and the sudden and steep increase in polarisation at the poles in all three wavelength bands.

The value of polarisation and the variation across latitude in the $V$ filter data are similar to those from other recent studies (Gorosabel et al. 2015). The difference in polarisation between the belts and zones is more prominent in the data presented here than in the work by Shalygina et al. (2008). This is especially made evident by the different wavelength bands used in this work and also the better viewing conditions in dataset IP5. Interestingly, the polarisation is consistently higher at the south pole than at the north pole. This appears to be a seasonal effect that has been observed and described before (Shalygina et al. 2008). Schmid et al. (2011) show from observations taken in 2003 that there is a higher polarisation across the south polar region than across the north polar region, which our results are also consistent with. The observations by Schmid et al. (2011) were taken during a similar seasonal period on Jupiter as ours, namely southern spring. While the obliquity angle of Jupiter is only $3.12^{\circ}$, Jupiter's orbit has a relatively large ellipticity (of 0.048 ) with a perihelion that almost coincides with the northern summer solstice, thus enhancing hemispherical differences in insolation. Our observations were carried out during the end of the southern winter season and the beginning of the southern spring, where the southern polarisation is still higher than the northern polarisation.

Figure 14 shows latitudinal plots of $P_{\mathrm{L}}$ along with latitudinal cuts of the total flux for dataset IP5. These plots show the greater sensitivity of the polarisation to variations across the disk of Jupiter. Flux measurements alone can reveal differences in the cloud type and structure, but the inclusion of polarimetric measurements gives a complete description of the light scattered within the planetary atmosphere. This is an important result not just for solar system planetary atmospheres, but also for exoplanets: polarimetric measurements coupled with radiative transfer models can constrain atmospheric properties much more than flux measurements alone can. The challenge of the greater sensitivity of polarisation data is that it makes fitting the data much harder than fitting total flux data. Section 7.3 discusses this in more detail and presents some sample results of models of the light reflected by the atmospheres of exoplanets.

Each imaging polarimetry dataset shows a wealth of detail and the same trends in polarisation across the disk of Jupiter with slight variations depending on the belt and zonal cloud regions. The variation in polarisation across the central part of the disk is more obvious in the $B$ filter observations; this appears to be due to a higher proportion of strongly polarising Rayleigh scattering from hydrogen, helium, and methane molecules. Table 1 shows that, for each dataset, the time difference between observations in each filter can be up to around $20 \mathrm{~min}$. This corresponds to approximately $1 / 30$ th of Jupiter's rotation period $(9.9 \mathrm{~h})$ or $12 \mathrm{deg}$ of rotation, ultimately meaning that a different region of the planet was sampled, which could be the cause of some of the differences in flux and polarisation between filters. The results compare favourably with previous studies, such as Shalygina et al. (2008), who also found that from the average of 15 datasets taken over $23 \mathrm{yr}$, the southern polar region of Jupiter was more strongly polarised at blue wavelengths than the northern polar region. Schmid et al. (2011) also observed this from spectropolarimetric observations with the slit positioned at the polar regions.

\section{Theoretical modelling}

In order to interpret the data presented in the previous section, numerical models of the polarisation of the reflected light were carried out via an adding-doubling radiative transfer code. The modelling was achieved by considering the atmosphere of Jupiter as constructed from locally plane parallel, horizontally homogeneous layers with a pressure profile as in Stam et al. (2004). Each layer in the atmosphere contains hydrogen, helium, and methane with some layers also containing clouds and hazes of varying thickness. The microphysical properties of the cloud and haze particles were varied, along with the height in the atmosphere of the different layers to try to reproduce the observed variation of polarisation across the different belts and zones of Jupiter. The modelling was carried out for spherical cloud and haze particles, described by Mie theory, and with fractal aggregate particles used to model the polar haze particles, as in Karalidi et al. (2013).

\subsection{The radiative transfer code}

The radiative transfer code we use to simulate our observations in order to characterise Jupiter's atmosphere is an addingdoubling algorithm similar to that used by Karalidi et al. (2013). This code is based on the description by de Haan et al. (1987) and fully includes polarisation for all orders of scattering. Given a vertically layered model atmosphere with scattering and/or absorbing gas molecules, cloud, and/or haze particles, bounded below by a black or reflecting surface, the code computes the Stokes vector (see Eq. (1)) of the reflected sunlight for the user defined illumination and viewing angles. 
The user defined illumination (incidence) and viewing (emergence) angles are the following: $\theta_{0}$ is the angle between the incident sunlight and local vertical direction (the solar zenith angle); $\theta$ is the angle between the reflected sunlight and local vertical direction; and $\phi-\phi_{0}$ is the azimuthal angle between the direction of propagation of the incident sunlight and reflected sunlight, measured in the local horizontal plane. Since our atmospheric layers are horizontally homogeneous, only the differential azimuthal angle is relevant. Given a pixel on the planet and phase angle, we compute the local values for $\theta_{0}, \theta$, and $\phi-\phi_{0}$. The locally reflected flux vector (defined with respect to the local meridian plane, which contains the local zenith and the direction towards the observer), $\boldsymbol{F}$, is calculated as follows (see Hansen \& Travis 1974):

$$
\boldsymbol{F}\left(\mu, \mu_{0}, \phi-\phi_{0}\right)=\mu_{0} \mathbf{R}\left(\mu, \mu_{0}, \phi-\phi_{0}\right) \boldsymbol{F}_{0}
$$

where $\boldsymbol{F}_{0}$ represents the vector of the incident (unpolarised) stellar or solar flux, and $\mathbf{R}$ represents the $4 \times 4$ local planetary scattering matrix. The values $\mu_{0}=\cos \theta_{0}\left(0^{\circ} \leq \theta_{0} \leq 90^{\circ}\right)$ and $\mu=\cos \theta$ $\left(0^{\circ} \leq \theta \leq 90^{\circ}\right)$. The models used all assume incident unpolarised light from the star or the Sun, meaning that the incident vector is always $\boldsymbol{F}_{0}=\left[F_{0}, 0,0,0\right]$, where $F_{0}$ is the total incident solar or stellar flux measured perpendicular to the direction of incidence divided by $\pi$ (see Hansen \& Travis 1974). Since the incident light is unpolarised, only the first column of the $4 \times 4$ planetary reflection matrix $\mathbf{R}$ is required, meaning Eq. (11) becomes

$$
\boldsymbol{F}\left(\mu, \mu_{0}, \phi-\phi_{0}\right)=\mu_{0} \mathbf{R}_{1}\left(\mu, \mu_{0}, \phi-\phi_{0}\right) \boldsymbol{F}_{0},
$$

where $\mathbf{R}_{1}$ represents the first column of the planetary reflection matrix. Given a (local) model atmosphere, we calculate $\mathbf{R}_{1}$ for the given local illumination and viewing geometries with our adding-doubling algorithm. Because we ignore the circular polarisation in our computations, we only compute the first three elements of $\mathbf{R}_{1}$. As mentioned before, the reference plane for the locally computed Stokes parameters $Q$ and $U$ is the local meridian plane. The degree of linear polarisation of the reflected light (see Eq. (3)) is independent of the choice of reference plane.

Because we are mostly interested in computing and comparing the normalised reflected fluxes and the degree of polarisation, we do not have to take the solar spectrum and solar flux that is incident on Jupiter into account. Furthermore, the distance between Jupiter and Earth and the angular size of Jupiter are irrelevant for our modelling.

\subsection{The model atmospheres}

The model atmospheres used in the numerical simulations consist of stacked horizontally homogeneous layers in locally planeparallel layers, which contain gas molecules and optionally contain cloud or haze particles. The model atmospheres are bounded below by black, homogenous surfaces; the optical thickness of the atmosphere precludes an influence of the surface albedo. A representation of this is shown in Fig. 15.

The radiative transfer calculations require knowledge for each atmospheric layer of the optical thickness, $b$, the single scattering albedo, $a$, and the single scattering matrix, $\mathbf{S}_{\text {sca }}$, of the mixture of molecules, cloud, and/or haze particles. The optical thickness of an atmospheric layer, $b$, is given by the sum of the molecular, cloud, and/or haze extinction optical thicknesses as in Stam (2008).

The Rayleigh scattering optical thickness is higher in the $B$ filter, decreasing with approximately $\lambda^{-4}$ to its lowest value

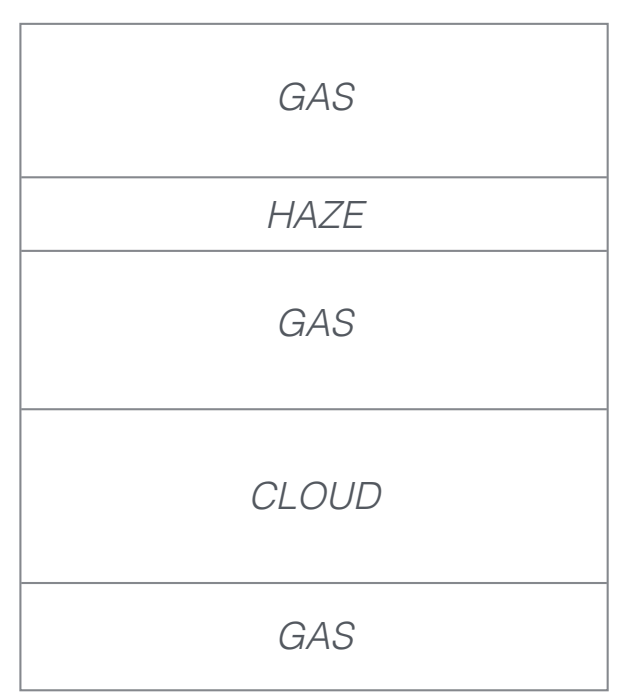

Fig. 15. Basic schematic of the model atmospheric layers. Gas layers consist of gaseous molecules, the scattering properties of which are described by Rayleigh scattering theory. The cloud and haze layers contain gaseous molecules and larger particles that fall under the Mie scattering regime. Pressure increases with decreasing height, so the clouds are at a higher pressure (lower altitude) than the haze. The clouds are located in the troposphere of Jupiter (with a cloud-top height varying between 0.56 bar to 1.778 bar) and the haze is located in the stratosphere between 0.10 bar and 0.13 bar

in the $R$ filter. The molecular scattering optical thicknesses of the individual atmospheric layers, $b_{\text {sca }}^{\mathrm{m}}$, depend on the molecular column density (i.e. the number of molecules per $\mathrm{m}^{2}$ ), refractive index of the gas, and depolarisation factor of the molecular mixture, for which we use the typical value for a mostly hydrogen containing atmosphere (see Hansen \& Travis 1974). The molecular column density is a function of the ambient temperature and pressure. The model results presented in this work were calculated using a 20 layer model atmosphere with data taken from Lindal (1992) and supplemented from 1 to 5.623 bars by data taken from West et al. (1986). We assume the atmospheric layers are in hydrostatic equilibrium. The wavelength region considered in all of the models we used is from 0.4 to $1.0 \mu \mathrm{m}$, and in this region the main absorbing gas in the Jovian atmosphere is methane $\left(\mathrm{CH}_{4}\right)$, which has an atmospheric mixing ratio of $0.18 \%$. The $\mathrm{CH}_{4}$ absorption cross sections of Karkoschka (1994) were used for our models.

The cloud and haze (except those of the fractal particles) particle properties used are calculated with a Mie algorithm, as described by de Rooij \& van der Stap (1984), and are calculated using a standard size distribution as defined by Hansen $\&$ Travis (1974), as follows:

$n(r)=C r^{\left(1-3 v_{\text {eff }}\right) / v_{\text {eff }}} \mathrm{e}^{-r / v_{\text {eff }} r_{\text {eff }}}$

where $C$ is a normalisation constant, $n(r) \mathrm{d} r$ is the number of particles per unit volume that have radii between $r$ and $r+\mathrm{d} r, r_{\text {eff }}$ is the effective radius, and $v_{\text {eff }}$ is the effective variance. The units of $r_{\text {eff }}$ are microns, whilst $v_{\text {eff }}$ is dimensionless.

The single scattering albedo of the mixture of gas molecules, and either cloud or haze particles, is given by

$a(\lambda)=\frac{b_{\mathrm{sca}}^{\mathrm{m}}(\lambda)+b_{\mathrm{sca}}^{\mathrm{a}}(\lambda)}{b^{m}(\lambda)+b^{a}(\lambda)}$, 

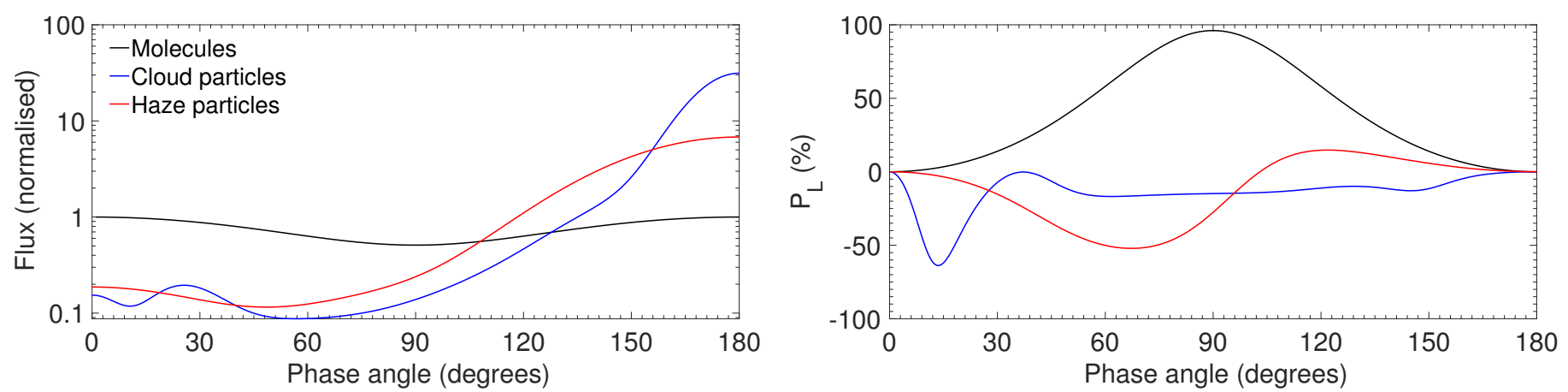

Fig. 16. Single scattering flux and linear polarisation of the cloud and haze particles at $550 \mathrm{~nm}$ along with Rayleigh scattering curves. The phase angle, $\alpha$, is $180^{\circ}-\Theta$, where $\Theta$ is the single scattering angle.

and the scattering matrix of the mixture (see Hovenier et al. 2004) is calculated as

$\mathbf{S}_{\mathrm{sca}}(\lambda)=\frac{b_{\mathrm{sca}}^{\mathrm{m}}(\lambda) \mathbf{S}_{\mathrm{sca}}^{\mathrm{m}}(\lambda)+b_{\mathrm{sca}}^{\mathrm{a}}(\lambda) \mathbf{S}_{\mathrm{sca}}^{\mathrm{a}}(\lambda)}{b_{\mathrm{sca}}^{\mathrm{m}}(\lambda)+b_{\mathrm{sca}}^{\mathrm{a}}(\lambda)}$,

where $\mathbf{S}_{\mathrm{sca}}^{\mathrm{m}}$ and $\mathbf{S}_{\mathrm{sca}}^{\mathrm{a}}$ are the scattering matrices of the molecules and either the cloud or haze particles, respectively. All of the scattering matrices depend on the wavelength $\lambda$, but are also functions of the scattering angle, $\Theta$ (with $\Theta=180^{\circ}-\alpha$, where $\alpha$ is the planetary phase angle).

The physical properties of the clouds and hazes of Jupiter and gas giants, in general, vary with time and location on the planet. Thermodynamic models of the atmosphere of Jupiter indicated that the cloud layers in the upper troposphere should consist of ammonia ice (see Sato \& Hansen 1979; Simon-Miller et al. 2001; de Pater \& Lissauer 2001). Data from the Galileo and Cassini missions, however, indicated that ammonia ice clouds only cover small regions of the planet (see Baines et al. 2002; Wong et al. 2004). Atreya et al. (2005) put forward a solution to this apparent contradiction: that the ammonia ice is coated by hydrocarbon haze particles condensing from the stratosphere, thus meaning that only the highest, or freshest, ammonia ice clouds would show identifiable spectral features, also depending on the size and shapes of the ice crystals. Recent results on the distribution of ammonia in the atmosphere of Jupiter are discussed by de Pater et al. (2016). Section 7 presents the results of our modelling.

\section{Modelling of the observations}

Several cloud particle types were used in test models of the Jovian atmosphere. The best solution found from our model fitting was a model atmosphere containing both an optically thick tropospheric cloud layer, along with an overlying stratospheric haze layer. The cloud particle type chosen was one with $r_{\text {eff }}=0.50 \mu \mathrm{m}$, and $v_{\text {eff }}=0.05$ with a refractive index of 1.42 and an imaginary part of 0.015 . A haze with $r_{\text {eff }}=0.2 \mu \mathrm{m}$ was found to give the closest solution with a narrow size distribution of $v_{\text {eff }}=0.01$. The refractive index of the haze was chosen to be 1.50 (Stoll 1980) with an imaginary part of 0.001. Both the size and the refractive index of the haze is consistent with that found by Stoll (1980), who modelled Pioneer observations of the polarisation of Jupiter and attempted to constrain the cloud and haze particle properties. Figure 16 shows the single scattering flux and polarisation for the cloud and haze particles. The parameter $P_{\mathrm{L}}$ has negative values because here $P_{U}=0$, so $P_{\mathrm{L}}$ has been set equal to $-P_{Q}$, thus the positive (negative) values of $P_{\mathrm{L}}$

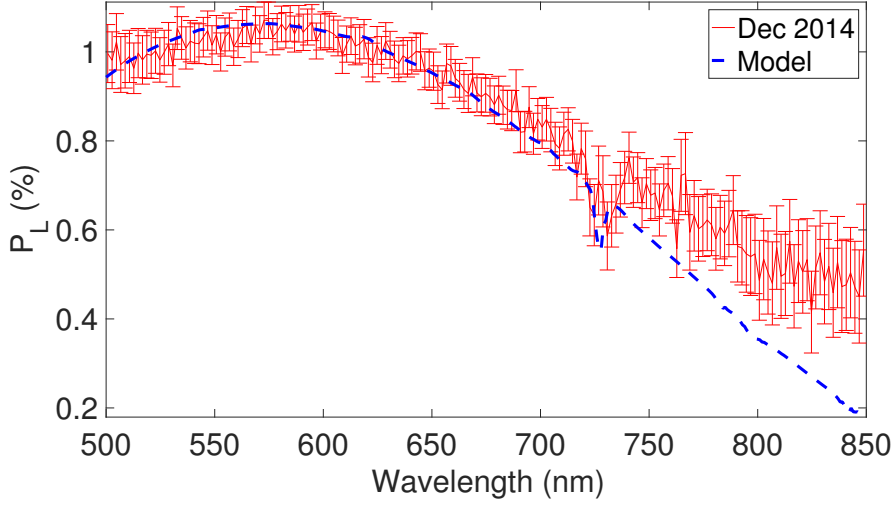

Fig. 17. Model fit to the December 2014 data.

in Fig. 16 indicate polarisation that is perpendicular (parallel) to the scattering plane.

\subsection{Spectropolarimetry models}

The best fitting models found for the spectropolarimetric data are shown in Figs. 17 and 18. The data are shown as solid lines, with error bars, and the models overlaid as dashed lines. The slit for each observation covers a wide range of regions in Jupiter's atmosphere; there are contributions from the belts, zones, and polar regions. The model fits to the spectropolarimetric data only consider a single atmosphere model, effectively representing the atmospheric properties across the region sampled in the slit. The December 2014 model has an optically thick cloud particle layer at a cloud-top height of 1.0 bar with a diffuse haze layer from 0.13 to 0.10 bar. The cloud layer for the November 2015 model has a slightly higher vertical extent, extending up to 0.56 bar with a slightly thicker haze layer than the December 2014 model at the same altitude. A fit that replicated both the continuum polarisation and the polarisation across the methane absorption bands for the November 2015 dataset proved to be challenging to find, so the fit that gives the best approximation to the continuum is shown.

The models of the spectropolarimetric data mostly replicate the continuum polarisation over the wavelength range observed, and there is no inconsistency between the blue wavelengths and longer wavelengths, although further observations at bluer wavelengths would be required to fully investigate this. The fit to the November 2015 dataset (see Fig. 18) can be seen to deviate from the observed values of $P_{\mathrm{L}}$ at shorter wavelengths; this is similar to what is seen in the models of the imaging polarimetry data (see Fig. 19). The spectropolarimetric 


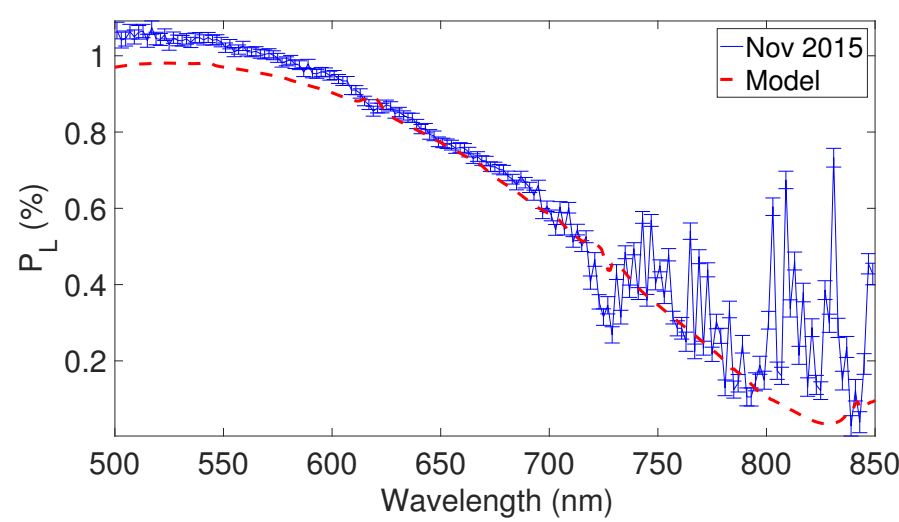

Fig. 18. Model fit to the November 2015 data.

observations encompassed a wide range of atmospheric properties along the regions sampled in the slit (see Fig. 2), so it is to be expected that there are different particle types giving a contribution to the overall polarisation. The methane absorption was successfully modelled for the December 2014 dataset, but a model consistent with both the absorption band depth and continuum polarisation could not be achieved for the November 2015 dataset.

\subsection{Models of the latitudinal profiles}

The modelling of the imaging data was carried out by fitting models using a particle type identical to those for the spectropolarimetry models. Interestingly, small spherical particles that scatter according to Mie theory appeared to be too depolarising and unable to fit the vast increase in polarisation in the polar regions. We thus decided to use fractal aggregate haze particles to model the polarisation at the poles, which are the same haze particles as used by Karalidi et al. (2013). These particles consist of 94 spherical monomers with each individual monomer having a radius of approximately $0.035 \mu \mathrm{m}$, and the volume-equivalent sphere radius of the entire particle is $0.16 \mu \mathrm{m}$. A refractive index of $1.5+0.001 \mathrm{i}$ was used, which corresponds to the optical properties of benzene; Friedson et al. (2002) suggest this may be present at the polar regions of Jupiter. The fractal aggregates were found to give a slightly higher polarisation than individual spherical haze particles. Optically thick layers of smaller haze particles were trialled in the models, but were not found to give sufficiently high values of polarisation to match the observational data. The optical thickness of the haze and cloud altitude were varied over latitude to reproduce the observed polarisation values. Table 2 gives the particle properties and the cloud and haze height and optical thickness of the final model used for each dataset.

Models of the polarisation across the disk of Jupiter are shown in Fig. 19 along with the observational data for comparison. We are able to reproduce the general variations in polarisation that are observed along central meridian of Jupiter, in particular, the high polarisation values in the polar regions and the differences between the belts and zones. These models have the same cloud and haze particle types as for the spectropolarimetry data. Because of the inconsistency in the latitudinal variations between the three filters (such as an inversion of polarisation), a model that reproduces the polarisation changes across the central meridian in all three bands has not yet been achieved. This is perhaps due to the limitations in the particle types that are used, since these are not fully understood. It is not just in the polar haze that non-spherical particles could be present, but particles in the zones and/or belts could also contain particles of different shapes, such as ice crystals. Models of the latitudinal variation could be produced for the $V$ and $R$ filters, but these are not consistent with the $B$ filter data. The reason for the different behaviour of the polarisation in the blue could be due to absorption, either by gas or a type of cloud or haze particle. Jupiter is known to have an as yet unknown UV absorber (see Karkoschka 1994), and a more detailed investigation of this will be the subject of a future paper. The same challenge, mainly of finding a fit with wavelength, has presented itself with the modelling of cometary data, with a potential solution of using non-spherical particles being put forward by some groups (see Kolokolova \& Kimura 2010; Kolokolova \& Mackowski 2012). Depending on the sizes of such non-spherical particles, the effects of the non-sphericity could be strongest at the shorter wavelengths.

The models used in this study use spherical particles generated from Mie theory to simulate ammonia ice particles as the main tropospheric Jovian clouds. The type of haze chosen for the lower latitudes was very arbitrary since not much is actually known about these types of particles, which are known to reside in the tropospheric and stratospheric regions of the planet's atmosphere, but was found to be consistent with the haze used by Stoll (1980). The model fits had to be both consistent with the wavelength and phase angle. Polarisation of Jupiter has occasionally been observed at phase angles close to $90^{\circ}$, for example by Cassini as it passed by the planet on its way to Saturn (Vasavada \& Showman 2005), and the same trends have been shown as in this study, namely, a relatively high value at the poles and a much lower value in the equatorial regions. However, it has been noted before that particles of any shape appear to be insufficient to fully characterise the Jovian atmosphere because of the limited phase angle range observable from Earth (Dlugach \& Mishchenko 2005). Indeed, a wider phase angle range, and with that a better shot at a full characterisation, is only achievable from a Jupiter-orbiting spacecraft or a fly-by mission.

\subsection{Models of signals from exoplanets}

Next, we investigated the flux and polarisation signals of Jupiter if it were an exoplanet. The flux and polarisation of an exoplanet would be received by a detector as an integrated value from the visible, illuminated part of the planetary disk, depending on the locally reflected starlight across the disk and thus on variations in atmospheric properties across the disk. Here we present some sample models of signals from gaseous exoplanets, based on our model fits to the Jupiter data, to demonstrate both the effect of differences across the disk and the added information that can be gleaned from polarimetry.

Similarly to Stam et al. (2004), we present models of the flux and polarisation of light reflected by Jupiter-like exoplanets of varying configuration. The models shown here are slightly different than those in Stam et al. (2004) because we use a different atmospheric configuration and different cloud and haze particles. Figure 20 shows models of both the flux and polarisation for three model atmospheres. The phase angle for each model is $90^{\circ}$, and the model cloud and haze particles are the same as used for the model interpretation of the observational data. Figure 21 shows the same models as a function of phase angle at a wavelength of $550 \mathrm{~nm}$. The neutral points in the polarisation phase function are very specific for the atmospheric scatterers. The distance to the system and the size of the planet are needed to calculate absolute fluxes, and these parameters can be difficult to 
Table 2. Model parameters.

\begin{tabular}{|c|c|c|c|c|c|}
\hline Data & Latitude (pixels) & Cloud-top pressure (bar) & Haze-top pressure (bar) & $b^{\mathrm{c}}$ & $b^{\mathrm{h}}$ \\
\hline IP1 & $4-12$ & 1.000 & 0.133 (fractals) & 50.0 & 2.000 \\
\hline IP1 & $13-54$ & 1.000 & 0.133 & 50.0 & 0.150 \\
\hline IP1 & $55-66$ & 0.562 & 0.133 & 50.0 & 0.010 \\
\hline IP1 & $67-85$ & 1.778 & 0.133 & 50.0 & 0.100 \\
\hline IP1 & $86-96$ & 0.562 & 0.133 & 50.0 & 0.100 \\
\hline IP1 & $97-111$ & 0.562 & $\mathrm{n} / \mathrm{a}$ & 50.0 & $\mathrm{n} / \mathrm{a}$ \\
\hline IP1 & $112-123$ & 1.778 & $\mathrm{n} / \mathrm{a}$ & 50.0 & $\mathrm{n} / \mathrm{a}$ \\
\hline IP1 & $124-135$ & 0.562 & 0.133 & 50.0 & 0.100 \\
\hline IP1 & $136-143$ & 0.562 & 0.133 & 50.0 & 0.125 \\
\hline IP1 & $144-158$ & 0.562 & 0.133 & 50.0 & 0.150 \\
\hline IP1 & $159-179$ & 1.000 & 0.133 & 50.0 & 0.150 \\
\hline IP1 & $180-184$ & 1.000 & 0.133 (fractals) & 50.0 & 2.000 \\
\hline IP2 & $4-9$ & 1.000 & 0.133 (fractals) & 50.0 & 3.000 \\
\hline IP2 & $10-19$ & 1.778 & 0.133 & 50.0 & 0.100 \\
\hline IP2 & $20-55$ & 1.000 & 0.133 & 50.0 & 0.150 \\
\hline IP2 & $56-68$ & 0.562 & 0.133 & 50.0 & 0.125 \\
\hline IP2 & $69-87$ & 1.778 & $\mathrm{n} / \mathrm{a}$ & 50.0 & $\mathrm{n} / \mathrm{a}$ \\
\hline IP2 & $88-164$ & 0.562 & 0.133 & 50.0 & 0.125 \\
\hline IP2 & $165-182$ & 1.778 & 0.133 & 50.0 & 0.100 \\
\hline IP2 & $183-184$ & 1.000 & 0.133 (fractals) & 50.0 & 3.000 \\
\hline IP3 & $4-9$ & 1.000 & 0.133 (fractals) & 50.0 & 3.000 \\
\hline IP3 & $10-19$ & 1.778 & 0.133 & 50.0 & 0.100 \\
\hline IP3 & $20-25$ & 1.778 & $\mathrm{n} / \mathrm{a}$ & 50.0 & $\mathrm{n} / \mathrm{a}$ \\
\hline IP3 & $26-45$ & 1.000 & $\mathrm{n} / \mathrm{a}$ & 50.0 & $\mathrm{n} / \mathrm{a}$ \\
\hline IP3 & $46-67$ & 0.562 & 0.133 & 50.0 & 0.125 \\
\hline IP3 & $68-85$ & 1.778 & 0.133 & 50.0 & 0.100 \\
\hline IP3 & 86-109 & 0.562 & 0.133 & 50.0 & 0.125 \\
\hline IP3 & $110-118$ & 1.778 & $\mathrm{n} / \mathrm{a}$ & 50.0 & $\mathrm{n} / \mathrm{a}$ \\
\hline IP3 & $119-160$ & 0.562 & 0.133 & 50.0 & 0.125 \\
\hline IP3 & $161-178$ & 1.778 & 0.133 & 50.0 & 0.100 \\
\hline IP3 & $179-184$ & 1.000 & 0.133 (fractals) & 50.0 & 3.000 \\
\hline IP4 & $4-6$ & 1.000 & 0.133 (fractals) & 50.0 & 3.000 \\
\hline IP4 & $7-15$ & 1.778 & 0.133 & 50.0 & 0.100 \\
\hline IP4 & $16-37$ & 1.000 & 0.133 & 50.0 & 0.150 \\
\hline IP4 & $38-43$ & 1.000 & 0.133 & 50.0 & 0.100 \\
\hline IP4 & $44-54$ & 1.000 & 0.133 & 50.0 & 0.300 \\
\hline IP4 & $55-72$ & 1.000 & 0.133 & 50.0 & 0.150 \\
\hline IP4 & $73-82$ & 1.000 & 0.133 & 50.0 & 0.300 \\
\hline IP4 & $83-104$ & 1.000 & 0.133 & 50.0 & 0.150 \\
\hline IP4 & $105-120$ & 0.562 & 0.133 & 50.0 & 0.200 \\
\hline IP4 & $121-126$ & 1.778 & 0.133 & 50.0 & 0.100 \\
\hline IP4 & $127-130$ & 1.000 & 0.133 (fractals) & 50.0 & 3.000 \\
\hline IP5 & $3-5$ & 1.000 & 0.133 (fractals) & 50.0 & 3.000 \\
\hline IP5 & $6-21$ & 1.778 & 0.133 & 50.0 & 0.100 \\
\hline IP5 & $22-28$ & 1.778 & 0.133 & 50.0 & 0.175 \\
\hline IP5 & $29-49$ & 1.000 & 0.133 & 50.0 & 0.225 \\
\hline IP5 & $50-68$ & 1.778 & 0.133 & 50.0 & 0.200 \\
\hline IP5 & $69-89$ & 1.778 & 0.133 & 50.0 & 0.100 \\
\hline IP5 & $90-97$ & 1.778 & 0.133 & 50.0 & 0.200 \\
\hline IP5 & $98-114$ & 1.000 & 0.133 & 50.0 & 0.225 \\
\hline IP5 & $115-128$ & 1.778 & 0.133 & 50.0 & 0.175 \\
\hline IP5 & $129-145$ & 1.778 & 0.133 & 50.0 & 0.100 \\
\hline IP5 & $146-148$ & 1.000 & 0.133 (fractals) & 50.0 & 3.000 \\
\hline
\end{tabular}

Notes. The values of $b^{\mathrm{c}}$ and $b^{\mathrm{h}}$ are those defined at $550 \mathrm{~nm}$. Unless indicated otherwise, the particle parameters for both the cloud and haze are the same as those given in the main body of the text. 

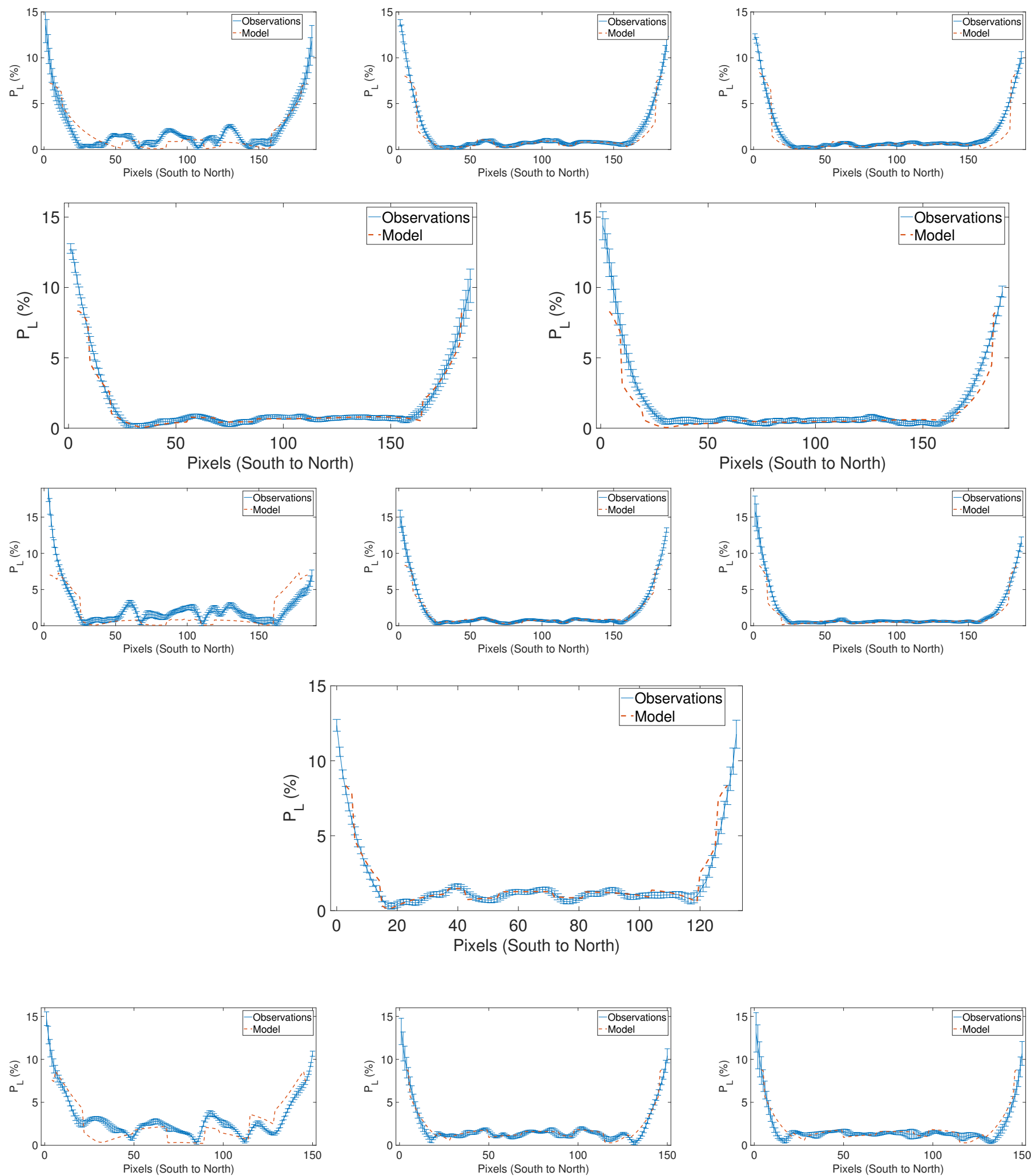

Fig. 19. Latitudinal models of the polarisation along Jupiter's central meridian, along with the data for comparison. From top to bottom: models of IP1, IP2, IP3, IP4, and IP5. Datasets IP1, IP3, and IP5 all have models shown in $B$ (left plot), $V$ (middle plot), and $R$ (right plot), IP2 has only models for $V$ (left plot), and $R$ (right plot), whilst IP4 has just models in the $V$ filter shown.

obtain. Since the degree of polarisation is a relative measure, it is independent of these quantities, thus one can glean atmospheric information from polarimetric measurements.

Model 1 has an atmosphere with no cloud or haze particles, only molecular gas. The continuum flux decreases with increasing wavelength because of the decrease in the molecular scattering optical thickness with wavelength. The continuum polarisation shows a different variation with wavelength than the flux, since a smaller Rayleigh scattering optical thickness results in less multiple scattering taking place, and multiple scattering 

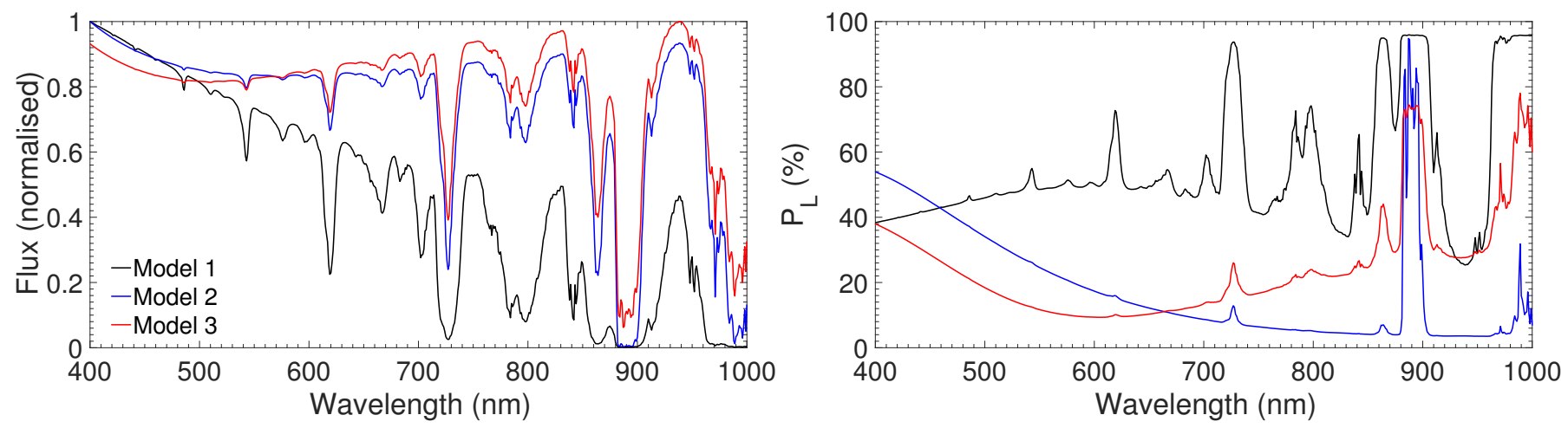

Fig. 20. Models of the flux and degree of linear polarisation of incident starlight reflected by three Jupiter-like exoplanets, for a phase angle of $90^{\circ}$. Model atmosphere 1 has only molecules, model 2 is similar to model 1, but with the addition of a tropospheric cloud layer, and model 3 is similar to model 2, with the inclusion of a stratospheric haze layer.
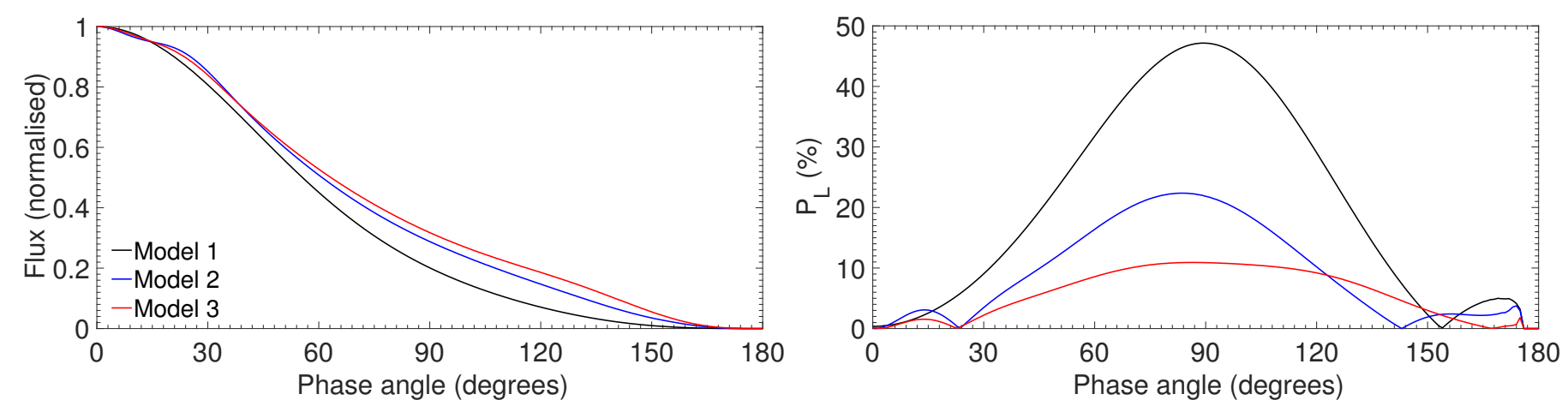

Fig. 21. Flux and degree of linear polarisation of the three model atmospheres as a function of the planetary phase angle for a wavelength of $550 \mathrm{~nm}$.

tends to lower the degree of polarisation of the reflected light. Increased absorption by $\mathrm{CH}_{4}$ decreases the amount of multiple scattering, resulting in high values of polarisation in the $\mathrm{CH}_{4}$ bands (Stam et al. 1999, 2004), similar to what has been observed by Schmid et al. (2011).

Model 2 has the same atmospheric configuration as model 1, but with the addition of a thick (cloud optical thickness $b^{\mathrm{c}}=50$ ) tropospheric cloud layer with a cloud-top pressure of $1.0 \mathrm{bar}$. At shorter wavelengths, the molecular scattering optical thickness of the gaseous atmosphere above the cloud layer is largest, which results in less light reaching the cloud layer; with increasing wavelength the contribution of light scattered by the cloud particles begins to dominate for both the flux and polarisation. The continuum polarisation decreases as a result of increased multiple scattering within the cloud layers, and also owing to the lower degree of polarisation of light scattered from the cloud particles (which can be seen clearly in Fig. 16). In the strong $\mathrm{CH}_{4}$ absorption band at $890 \mathrm{~nm}$, the flux and polarisation of models 1 and 2 are very similar. This is because hardly any incident stellar light penetrates the atmosphere deep enough to reach the cloud layer because of the high molecular absorption optical thickness of the atmosphere above the cloud layer, meaning that most of the light is scattered in the highest atmospheric layers.

Model 3 contains the same cloud layer as model 2, with the addition of a stratospheric haze layer of haze optical thickness $b^{\mathrm{h}}=0.2$, at a haze-top pressure of 0.133 bar. The polarisation at larger wavelengths is higher for model 3 than model 2; this is due to a higher (absolute) degree of polarisation of light single scattered from the haze particle layer, as can be seen from Fig. 16 .
Since model atmosphere 3 contains a high altitude haze, the light that would be observed at wavelengths where $\mathrm{CH}_{4}$ absorbs would not only be mostly singly scattered light, but it would also carry the single scattering signature of the haze particles. Because light singly scattered by these particles has a low degree of polarisation at a scattering angle of $90 \mathrm{deg}$, the polarisation in the bands is low. The polarisation at bluer wavelengths is higher than that for similar models by Stam et al. (2004) because we use a higher value of the complex part of the refractive index.

\section{Discussion and summary}

Seven different polarimetric datasets from the planet Jupiter have been presented in this study, along with model fits. The data show consistent trends in the polarisation for each dataset with higher polarisation in the polar regions of Jupiter and generally a north-south polar asymmetry in the polarisation, as has also been found in previous studies. The spectropolarimetric datasets show a rise in polarisation at bluer wavelengths because of an increase in Rayleigh scattered light at these wavelengths, which is known to have a higher polarisation than light scattered from larger particles. The modelling of the spectropolarimetric data was able to reproduce the general shape with wavelength, along with the decrease in polarisation across the strong methane absorption band around $720 \mathrm{~nm}$ for the December 2014 dataset.

The model fits to the imaging polarimetry data reproduce the general shape of the polarisation curve across latitude for the $V$ and $R$ filters, but are not consistent with the $B$ filter data. The resolution of this could be to use different particle shapes, as spherical particles could be a relatively poor approximation to the shape of those present in the Jovian atmosphere. Increased 
absorption at bluer wavelengths could also play a role in the difference in polarisation in the $B$-band data. Another limiting factor, which has been noted before, is that to fully quantify the atmospheric constituents of Jupiter, a wider phase angle range of data should be used, as was the case for the pioneering study of the Venusian clouds by Hansen \& Hovenier (1974). An advantage of studying exoplanets is that the full phase angle range is accessible, depending on the orbital inclination of the planet. Model results for Jupiter-like exoplanets were presented, showing the larger values of polarisation at a phase angle of $90^{\circ}$ and the difference between atmospheres containing cloud and haze particles.

Recent advances in telescope and instrument technology have provided a novel opportunity with which polarimetric observations from ground-based telescopes can provide strong constraints on the scattering properties of planetary atmospheres. Data from a $1 \mathrm{~m}$ telescope can provide sufficient signal and instrumental precision to produce polarimetric maps, which corroborate data previously obtained from larger telescopes and more highly tested instruments. In order to further constrain the properties of the scattering particles in Jupiter's atmosphere, more observations at different phase angles and wavelengths are required. Unfortunately, the ESA JUICE mission to Jupiter does not include a polarimeter. Studying the scattering layers of planetary atmospheres in the solar system also has an important application for exoplanetary studies, since studying the solar system will ultimately lead us to be able to constrain models of observations of light reflected by the atmospheres of exoplanets.

Acknowledgements. We gratefully acknowledge financial support from the COST Action MP1104 "Polarisation as a tool to study the Solar System and beyond". We also acknowledge the support of the INAF PRIN programme in the construction of the Torino Polarimeter. Additionally, the authors gratefully acknowledge observing grant support from the Institute of Astronomy and Rozhen National Astronomical Observatory, Bulgarian Academy of Sciences.

\section{References}

Atreya, S. K., Wong, A. S., Baines, K. H., Wong, M. H., \& Owen, T. C. 2005, Planet. Space Sci., 53, 498

Bagnulo, S., Landolfi, M., Landstreet, J. D., et al. 2009, PASP, 121, 993

Baines, K. H., Carlson, R. W., \& Kamp, L. W. 2002, Icarus, 159, 74

Carlson, B. E., \& Lutz, B. L. 1989, NASA SP, 494, 289

de Haan, J. F., Bosma, P. B., \& Hovenier, J. W. 1987, A\&A, 183, 371

de Pater, I., \& Lissauer, J. J. 2001, in Planetary Sciences (Cambridge, UK Cambridge University Press), 544

de Pater, I., Sault, R. J., Butler, B., DeBoer, D., \& Wong, M. H. 2016, Science, 352,1198

de Rooij, W. A., \& van der Stap, C. C. A. H. 1984, A\&A, 131, 237

Devogèle, M., Cellino, A., Bagnulo, S., et al. 2017, MNRAS, 465, 4335

Dlugach, Z. M., \& Mishchenko, M. I. 2005, Sol. Syst. Res., 39, 102

Dlugach, J. M., \& Mishchenko, M. I. 2008, MNRAS, 384, 64
Dollfus, A. 1957, Supplements aux Annales d'Astrophysique, 4, 3 Friedson, A. J., Wong, A.-S., \& Yung, Y. L. 2002, Icarus, 158, 389 Gehrels, T., Herman, B. M., \& Owen, T. 1969, AJ, 74, 190

Gorosabel, J., García Muñoz, A., Sánchez-Lavega, A., Hueso, R., \& Pérez Hoyos, S. 2015, in Highlights of Spanish Astrophysics VIII, Proc. of XI Sci. Meeting Spanish Astron. Soc., eds. A. J. Cenarro, F. Figueras, C. HernándezMonteagudo, J. Trujillo Bueno, \& L. Valdivielso, 868

Hall, J. S., \& Riley, L. A. 1976, Icarus, 29, 231

Hansen, J. E., \& Hovenier, J. W. 1974, J. Atm. Sci., 31, 1137

Hansen, J. E., \& Travis, L. D. 1974, Space Sci. Rev., 16, 527

Hovenier, J. W., \& van der Mee, C. V. M. 1983, A\&A, 128, 1

Hovenier, J. W., van der Mee, C., \& Domke, H., eds. 2004, Transfer of polarized light in planetary atmospheres: basic concepts and practical methods, Astrophysics and Space Science Library, 318

Ingersoll, A. P. 1976, Icarus, 29, 245

Ingersoll, A. P., Dowling, T. E., Gierasch, P. J., et al. 2004, Dynamics of Jupiter's atmosphere, eds. F. Bagenal, T. E. Dowling, \& W. B. McKinnon, 105

Jockers, K., Credner, T., Bonev, T., et al. 2000, Kinematika i Fizika Nebesnykh Tel Supplement, 3, 13

Karalidi, T., Stam, D. M., \& Guirado, D. 2013, A\&A, 555, A127

Karkoschka, E. 1994, Icarus, 111, 174

Kemp, J. C., Henson, G. D., Steiner, C. T., \& Powell, E. R. 1987, Nature, 326, 270

Kemp, J. C., \& Wolstencroft, R. D. 1971, Nature, 232, 165

Kolokolova, L., \& Kimura, H. 2010, Earth, Planets, and Space, 62, 17

Kolokolova, L., \& Mackowski, D. 2012, J. Quant. Spectr. Rad. Transf., 113, 2567

Lindal, G. F. 1992, AJ, 103, 967

Lyot, B. 1929, Annales de l'Observatoire de Paris, Section de Meudon, 8, 1

Mishchenko, M. I. 1990, Icarus, 84, 296

Morozhenko, A. V., \& Yanovitskii, E. G. 1973, Icarus, 18, 583

Oliva, E. 1997, A\&AS, 123

Pernechele, C., Abe, L., Bendjoya, P., et al. 2012, in SPIE Conf. Ser., 8446, $84462 \mathrm{H}$

Sato, M., \& Hansen, J. E. 1979, J. Atmos. Sci., 36, 1133

Schmid, H. M., Joos, F., Buenzli, E., \& Gisler, D. 2011, Icarus, 212, 701

Shalygina, O. S., Korokhin, V. V., Starukhina, L. V., et al. 2008, Sol. Sys. Res., 42,8

Simon-Miller, A. A., Banfield, D., \& Gierasch, P. J. 2001, Icarus, 154, 459

Smith, P. H., \& Tomasko, M. G. 1984, Icarus, 58, 35

Sromovsky, L. A., \& Fry, P. M. 2002, Icarus, 157, 373

Stam, D. M. 2008, A\&A, 482, 989

Stam, D. M., \& Hovenier, J. W. 2005, A\&A, 444, 275

Stam, D. M., De Haan, J. F., Hovenier, J. W., \& Stammes, P. 1999, J. Geophys. Res., 104, 16843

Stam, D. M., Hovenier, J. W., \& Waters, L. B. F. M. 2004, A\&A, 428, 663

Starodubtseva, O. M., Akimov, L. A., \& Korokhin, V. V. 2002, Icarus, 157, 419

Stoll, C. P. 1980, Ph.D. Thesis, Arizona University, USA

Vasavada, A. R., \& Showman, A. P. 2005, Rep. Progr. Phys., 68, 1935

West, R. A., Strobel, D. F., \& Tomasko, M. G. 1986, Icarus, 65, 161

West, R. A., Baines, K. H., Friedson, A. J., et al. 2004, in Jovian clouds and haze, eds. F. Bagenal, T. E. Dowling, \& W. B. McKinnon (Cambridge University Press), 79

West, R. A., Yanamandra-Fisher, P. A., \& Korokhin, V. 2015, in Polarimetry of Stars and Planetary Systems, eds. L. Kolokolova, J. Hough, \& A.-C. Levasseur-Regourd (Cambridge University Press), 320

Wiktorowicz, S. J., \& Stam, D. M. 2015, in Polarimetry of Stars and Planetary Systems, eds. L. Kolokolova, J. Hough, \& A.-C. Levasseur-Regourd (Cambridge University Press), 439

Wong, M. H., Bjoraker, G. L., Smith, M. D., Flasar, F. M., \& Nixon, C. A. 2004, Planet. Space Sci., 52, 385

Young, R. E. 2003, Astron. Rev., 47, 1 